\title{
Genome-wide characterization of JASMONATE-ZIM DOMAIN transcription repressors in wheat (Triticum aestivum L.)
}

\author{
Yukun Wang ${ }^{1,2+}$, Linyi Qiao ${ }^{3 \dagger}$, Jianfang Bai ${ }^{1,2 \dagger}$, Peng Wang ${ }^{1,2,4}$, Wenjing Duan ${ }^{1,2,5}$, Shaohua Yuan ${ }^{1,2}$,
} Guoliang Yuan ${ }^{1,2}$, Fengting Zhang ${ }^{1,2}$, Liping Zhang ${ }^{1,2^{*}}$ (D) and Changping Zhao ${ }^{1,2^{*}}$

\begin{abstract}
Background: The JASMONATE-ZIM DOMAIN (JAZ) repressor family proteins are jasmonate co-receptors and transcriptional repressor in jasmonic acid (JA) signaling pathway, and they play important roles in regulating the growth and development of plants. Recently, more and more researches on JAZ gene family are reported in many plants. Although the genome sequencing of common wheat (Triticum aestivum L.) and its relatives is complete, our knowledge about this gene family remains vacant.

Results: Fourteen JAZ genes were identified in the wheat genome. Structural analysis revealed that the TaJAZ proteins in wheat were as conserved as those in other plants, but had structural characteristics. By phylogenetic analysis, all JAZ proteins from wheat and other plants were clustered into 11 sub-groups (G1-G11), and TaJAZ proteins shared a high degree of similarity with some JAZ proteins from Aegliops tauschii, Brachypodium distachyon and Oryza sativa. The Ka/Ks ratios of TaJAZ genes ranged from 0.0016 to 0.6973 , suggesting that the TaJAZ family had undergone purifying selection in wheat. Gene expression patterns obtained by quantitative real-time PCR (qRT-PCR) revealed differential temporal and spatial regulation of TaJAZ genes under multifarious abiotic stress treatments of high salinity, drought, cold and phytohormone. Among these, TaJAZ7, 8 and 12 were specifically expressed in the anther tissues of the thermosensitive genic male sterile (TGMS) wheat line BS366 and normal control wheat line Jing411. Compared with the gene expression patterns in the normal wheat line Jing411, TaJAZ7, 8 and 12 had different expression patterns in abnormally dehiscent anthers of BS366 at the heading stage 6 , suggesting that specific up- or down-regulation of these genes might be associated with the abnormal anther dehiscence in TGMS wheat line.

Conclusion: This study analyzed the size and composition of the JAZ gene family in wheat, and investigated stress responsive and differential tissue-specific expression profiles of each TaJAZ gene in TGMS wheat line BS366. In addition, we isolated 3 TaJAZ genes that would be more likely to be involved in the regulation of abnormal anther dehiscence in TGMS wheat line. In conclusion, the results of this study contributed some novel and detailed information about JAZ gene family in wheat, and also provided 3 potential candidate genes for improving the TGMS wheat line.
\end{abstract}

Keywords: Anther dehiscence, Gene expression, JASMONATE-ZIM DOMAIN, Phylogenetic analysis, Thermo sensitive genic male sterile (TGMS)

\footnotetext{
*Correspondence: Ipzhang8@126.com; cp_zhao@vip.sohu.com

${ }^{\dagger}$ Equal contributors

${ }^{1}$ Beijing Engineering Research Center for Hybrid Wheat, Beijing Academy of

Agricultural and Forestry Sciences, Beijing 100097, China

Full list of author information is available at the end of the article
}

(c) The Author(s). 2017 Open Access This article is distributed under the terms of the Creative Commons Attribution 4.0 International License (http://creativecommons.org/licenses/by/4.0/), which permits unrestricted use, distribution, and reproduction in any medium, provided you give appropriate credit to the original author(s) and the source, provide a link to the Creative Commons license, and indicate if changes were made. The Creative Commons Public Domain Dedication waiver (http://creativecommons.org/publicdomain/zero/1.0/) applies to the data made available in this article, unless otherwise stated. 


\section{Background}

Jasmonic acid and its bioactivity derivatives are collectively known as Jasmonates (JAs). They play important roles in regulating the responses to biotic and abiotic stresses, such as herbivory, pathogen invasion, wounding, UV radiation and ozone stress in plants [1-8]. In addition, JAs also control various developmental processes in plants, viz. pollen maturation, anther dehiscence, embryo maturation, tendril coiling, tuber and trichome growth [9-12]. Therefore, JAs are regarded as moderators that mainly involved in regulating the defense reaction, inhibiting the photosynthesis and cell division, and keeping the balance between growth and defense in plants $[2,13,14]$.

In plants, JA signaling pathway is a very complex process involved in many genes or proteins $[15,16]$, and this pathway can be divided into three processes, including the biosynthesis and metabolism of signaling molecules, JA signal transduction and responses of downstream genes. Previous studies showed that JA signaling molecules, $\mathrm{SCF}^{\mathrm{COI1}}$ receptor complex, Jasmonate-ZIM (JAZ) domain repressor and transcription activator $\mathrm{MYC2}$ participate together and interact with each other in JA signaling pathway [17-21]. In the plant cells with a lower-concentration of JA, the transcription of JA response genes is restrained owing to the combination between JAZ protein and MYC2. Under stimulation, JA is compounded and accumulated in plant cells. High concentrations of JA can promote the combination between JAZ proteins and $\mathrm{SCF}^{\mathrm{COI}}$, and make JAZ proteins be ubiquitinated. Then the ubiquitinated protein is alternatively degraded by $26 \mathrm{~S}$ protease. Finally, the activity inhibition of transcription activator MYC2 is removed. Expression of JA response genes is activated subsequently [19]. It is known that JAZ proteins have a crucial effect on the JA signaling pathway and they are the linkers of JA signaling transduction.

The JAZ protein family is a member of TIFY transcription factor superfamily, and the family members have two conservative functional domains, TIFY (also known as ZIM) and Jas (also known as CCT_2) [22-24]. TIFY domain is usually consisted of 28 amino acids, which located in the $\mathrm{N}$-terminus of JAZ protein sequences, and its core sequence is TIF[F/Y]XG $[25,26]$. In JA signaling pathway, TIFY domain can mediate the interaction between JAZ protein and its co-suppressor, a NOVEL INTERACTOR of JAZ (NINJA), and then collectively restrict the JA signal transduction [27]. Jas domain is near the C-terminus of JAZ protein sequences, and its sequence is extremely conserved among the members of JAZ protein family, of which 10 amino acids are same or replaced in conservation. Its function is to mediate the direct combination between JAZ protein and $\mathrm{MYC} 2$ in JA signaling pathway and inhibit the transcriptional activity of MYC2 [28, 29], and then further restrict the expression of JA response genes.

As the connector between MYC2 and COI1, JAZ protein was firstly reported in 2007 [30, 31]. Afterwards, many studies about JAZ proteins have been conducted. To date, 12 and 15 JAZ genes have been found in Arabidopsis thaliana [13] and rice [32], respectively. According to previous studies, the $J A Z$ gene family has plenty of members, but each has a different biological function. For example, the protein encoded by OsJAZ9 is a regulator of JA signaling pathway and can regulate the salt tolerance of rice [33]. The expression of GhJAZ1 can be activated by GbWRKY1, so that it can strengthen the capacity for resistance against Verticillium dahlia in cotton [34]. Overexpression of modified AtJAZ1 (lack of Jas domain) is able to enhance host resistance to Spodoptera exigua in Arabidopsis [35]. Overexpressing the modified $O s J A Z s$ can cause the malformation of flower organ in rice [36]. According to the above researches, JAZ genes have important effects on regulating the adaptability to biotic and abiotic stresses and maintaining the normal development in plants.

Wheat is one of the most important crops in the world. The changes of climate and growing environments bring huge challenge to wheat production. Thus, use of molecular biology and genetics methods is an important approach for improving the stress tolerance and quality of wheat. Thermosensitive genic male sterile (TGMS) lines, such as BS366, are of particular significance in two-line hybrid system, which is more efficient in breeding [37], because the fertility of TGMS lines is strictly regulated by temperature [38-40]. Hybrid seed could be produced under sterile condition (TGMS line as maternal plant), while TGMS line itself could be reproduced under fertile condition [41]. In our early research, we found that the anther dehiscence in BS366 is abnormal, and its pollen can not fully spill out. This defective phenotype can be recovered via spraying MeJA in vitro [42]. So, we assumed that this phenotype is closely related to the synthesis and regulatory pathway of JA. Based on the current wheat genome sequencing data, we firstly identified the TaJAZ gene family, and analyzed its architectural features, evolutionary history and expression patterns in anther tissues in TGMS wheat line. The results presented in this study were expected to enrich our knowledge of $J A Z$ gene family in wheat, and provide the theoretical basis and novel candidate genes for improving and creating the male sterility wheat lines.

\section{Results}

Identification of JAZ repressor gene family in wheat

Based on the latest genome data of wheat, a hidden Markov model (HMM) search was carried out using the HMM profiles of the TIFY domain (Pfam accession No.: 
PF06200) and Jas domain (Pfam accession No.: PF09425) as queries against the local protein database. By retrieving the database, we detected 40 non-redundant sequences. Among these, 6 were removed because they lacked the typical TIFY or Jas domain. Finally, we obtained 34 full-length protein sequences (Table 1). The corresponding coding sequences and genome sequences were simultaneously isolated. The location of each $\mathrm{TaJAZ}$ gene was confirmed by aligning the wheat chromosome genomic sequences using BLASTn.
According to the information of phylogenetic relationship, 34 TaJAZ genes were clustered into 14 groups (Fig. 1a). The genes from different wheat sub-genomes and in the same group were regarded as different copies of each member of the TaJAZ gene family. Thus, we obtained the TaJAZ gene family including 14 members designated from 1 to 14 according to the naming convention, and the copies of each member were distinguished by subjoining the wheat sub-genome symbols A, B or D.

Table 1 JAZ gene family in wheat

\begin{tabular}{|c|c|c|c|c|c|}
\hline Genes & Sequence $I^{a}$ & Scaffold & Location & Length gDNA & Length AA \\
\hline TaJAZ1-A & Traes_2AS_A8CCC32D3.1 & 5193315 & 2AS:194518416-194520896 & 1657 & 232 \\
\hline TaJAZ1-B & Traes_2BS_2C79AE2DE.1 & 5245909 & 2BS:116670615-116671727 & 1628 & 232 \\
\hline TaJAZ1-D & Traes_2DS_C0C75D1D7.1 & 5342634 & 2DS:43488169-43490434 & 1645 & 232 \\
\hline TaJAZ2-B & Traes_2BL_0614A2B97.2 & 8023225 & 2BL:325113849-325118711 & 1568 & 152 \\
\hline TaJAZ3-A & Traes_2AL_6CBE19B87.2 & 6411971 & 2AL:249758979-249759677 & 3163 & 171 \\
\hline TaJAZ3-D & Traes_2DL_7DD4A39D4.2 & 9853109 & 2DL:75977386-75980215 & 2180 & 171 \\
\hline TaJAZ4-A & Traes_4AL_406C57F67.2 & 7068037 & 4AL:117374944-117379369 & 2615 & 213 \\
\hline TaJAZ4-B & Traes_4BS_8C20E76AA.2 & 4872575 & 4BS:8921165-8925871 & 2851 & 211 \\
\hline TaJAZ4-D & Traes_4DS_AFCEDDE67.2 & 2320697 & 4DS:39879093-39878219 & 4174 & 316 \\
\hline TaJAZ5-A & Traes_4AL_B6992AAA6.1 & 7095962 & 4AL:153112902-153117061 & 3902 & 271 \\
\hline TaJAZ5-B & Traes_4BS_ACD70539F.1 & 4890614 & 4BS:208712283-208716179 & 3616 & 270 \\
\hline TaJAZ5-D & Ta4dsLoc000428.2 & 1474230 & 4DS:5798624-5803047 & 3875 & 268 \\
\hline TaJAZ6-A & Traes_4AS_6EAA11AAD.2 & 5953686 & 4AS:31692130-31692495 & 539 & 138 \\
\hline TaJAZ6-D & Traes_4DL_E25D3DF01.1 & 14389161 & 4DL:102441958-102442530 & 546 & 182 \\
\hline TaJAZ7-A & Ta4asLoc008691.5 & 5960531 & 4AS:46818670-46818870 & 829 & 199 \\
\hline TaJAZ7-B & Traes_4BL_AA16A6065.2 & 7038768 & 4BL:296045108-296046696 & 932 & 234 \\
\hline TaJAZ7-D & Traes_4DL_7564D43A9.1 & 14466294 & 4DL:112478276-112479120 & 845 & 211 \\
\hline TaJAZ8-A & Ta5alLoc014221.1 & 2768335 & 5AL:143197395-143200628 & 1409 & 277 \\
\hline TaJAZ8-D & Ta4dILoc004501.1 & 14305556 & 4DL:120302264-120302430 & 1639 & 343 \\
\hline TaJAZ9-A & Traes_5AL_BB55F989A.2 & 2752881 & 5AL:106813685-106814577 & 701 & 152 \\
\hline TaJAZ9-B & Traes_5BL_7A6C3831E.1 & 10804653 & 5BL:50144832-50146521 & 1106 & 231 \\
\hline TaJAZ9-D & Traes_5DL_4186C5347.1 & 4498422 & 5DL:76465423-76467215 & 1096 & 231 \\
\hline TaJAZ10-A & Traes_5AL_BF3D7E764.1 & 7360 & 5AL:146353609-146353412 & 1269 & 285 \\
\hline TaJAZ10-B & Traes_5BL_1F38B9D05.1 & 10791567 & 5BL:76985189-76988406 & 2325 & 417 \\
\hline TaJAZ10-D & Traes_5DL_3A1F8C38E.1 & 4511779 & 5DL:80817088-80820136 & 2297 & 419 \\
\hline TaJAZ11-A & Traes_6AS_4106E8E28.1 & 4378986 & 6AS:35541777-35545250 & 3030 & 305 \\
\hline TaJAZ12-A & Traes_6AL_BC7FB0A99.1 & 5834167 & 6AL:183521365-183523426 & 1489 & 268 \\
\hline TaJAZ12-D & Traes_6DL_7024F5429.1 & 1374032 & 6DL:146803355-146805443 & 1508 & 268 \\
\hline TaJAZ13-A & Ta7asLoc006840.1 & 4189390 & 7AS:48401407-48404266 & 330 & 110 \\
\hline TaJAZ13-D1 & Traes_7DS_0B70FFE9A.1 & 3814476 & 7DS:31118625-31119606 & 330 & 110 \\
\hline TaJAZ13-D2 & Traes_7DS_C407F8C85.1 & 3930115 & 7DS:39462347-39463081 & 330 & 110 \\
\hline TaJAZ14-A & Traes_7AL_EA6F4FFDE.2 & 4463173 & 7AL:143335085-143340919 & 5523 & 257 \\
\hline TaJAZ14-B & Traes_7BL_7DC689032.1 & 6631476 & 7BL:205014054-205016812 & 2801 & 183 \\
\hline TaJAZ14-D & Traes_7DL_A5ECEDA95.1 & 3352851 & 7DL:203031373-203037757 & 5602 & 265 \\
\hline
\end{tabular}

${ }^{a}$ The sequence name beginning with Treas and TaLoc means download from the wheat genome Ensembl database and URGL database, respectively 


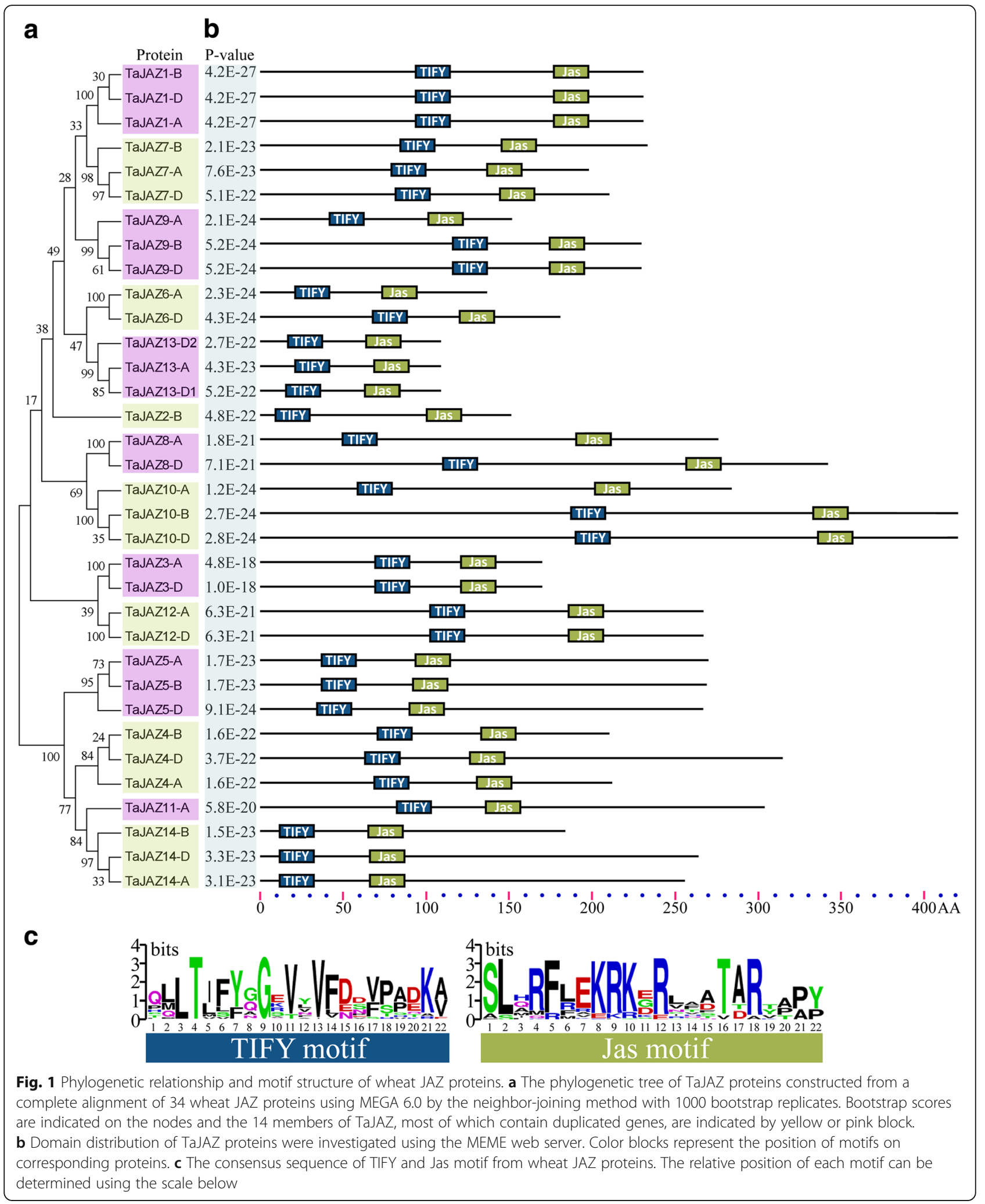

\section{Genome distribution of wheat $J A Z$ genes}

Among 34 wheat $J A Z$ genes, 13,8 and 13 were distributed in wheat sub-genomes A, B and D, respectively (Fig. 2).
None of TaJAZ gene copy was distributed on chromosomes $1 \mathrm{~A}, 3 \mathrm{~A}, 1 \mathrm{~B}, 3 \mathrm{~B}, 6 \mathrm{~B}, 1 \mathrm{D}$ and $3 \mathrm{D}$. Each of the 7 TaJAZ genes (TaJAZ1, 4, 5, 7, 9, 10 and 14) had three 


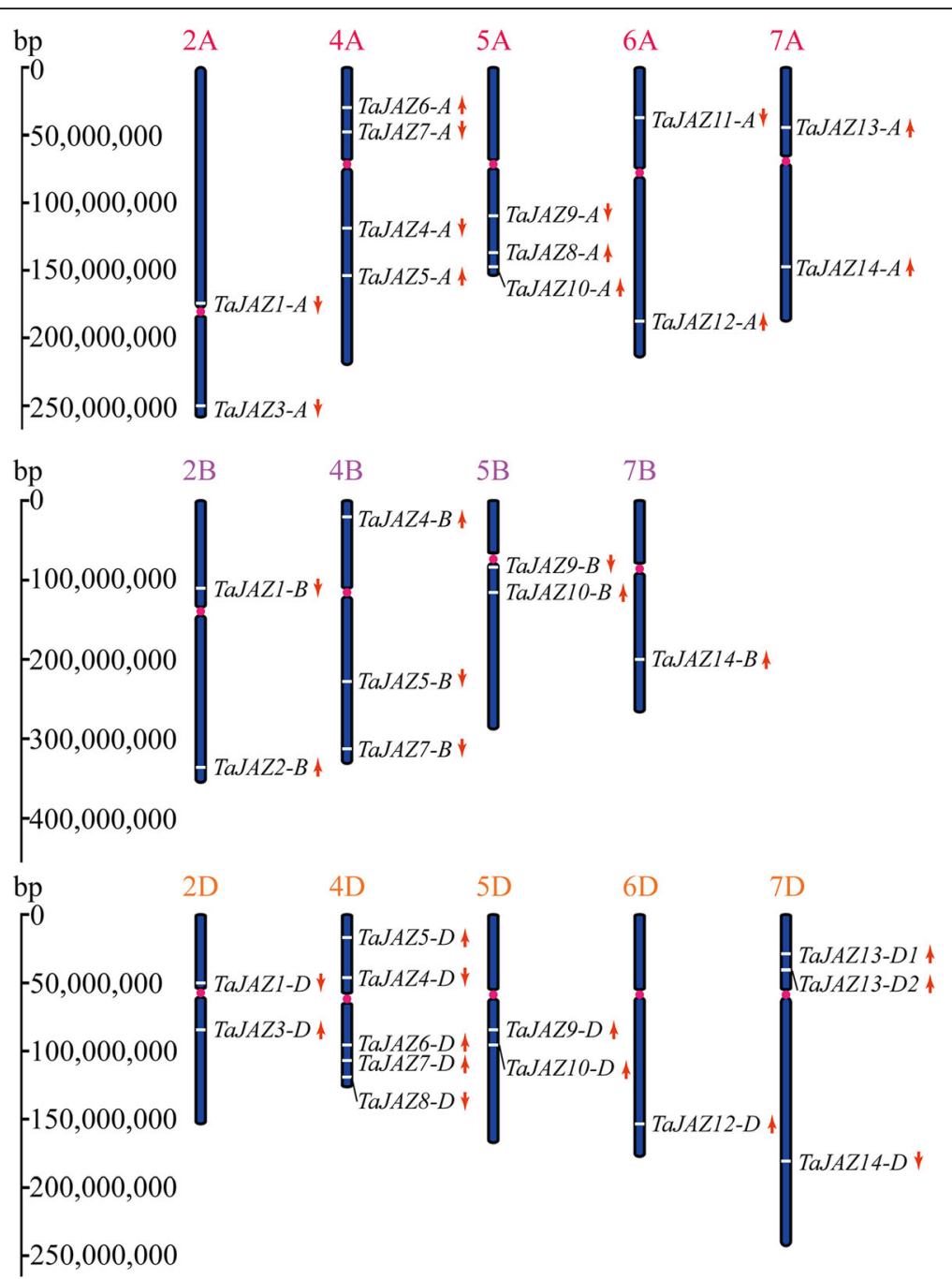

Fig. 2 Chromosome distribution of JAZ gene family in wheat. Red dots on the chromosomes indicate the position of centromeres. The red arrows next to gene names show the direction of transcription. The position of each gene could be estimated using the left scale

copies on group 2, 4, 5 and 7 chromosomes, respectively (Fig. 2). Four TaJAZ genes had two copies each including TaJAZ3-A/-D, TaJAZ6-A/-D, TaJAZ8-B/-D, and TaJAZ12$A /-D$ (Fig. 2). TaJAZ2 and TaJAZ11 had only one copy on chromosomes $2 \mathrm{~B}$ and $6 \mathrm{~A}$, respectively (Fig. 2). TaJAZ13 also had three copies, and TaJAZ13-A was found on chromosome 7A, TaJAZ13-D1 and -D2 were detected on chromosome 7D (Fig. 2). In addition, chromosome 4D had the highest number of five TaJAZ gene copies, whereas other chromosomes contained no more than four copies (Fig. 2).

Structure analysis of wheat $J A Z$ genes, and JAZ proteins In order to obtain more insights about the gene structural evolution, the exon-intron organization of wheat $J A Z$ genes was raveled by aligning the predicted coding sequences (CDS) against the corresponding genomic sequences using the online service GSDS. In the TaJAZ gene family, the number of exons ranged from 1 to 8 , and the number of introns ranged from 0 to 7 (Fig. 3). Among 12 TaJAZ genes containing two or three copies, 8 (TaJAZ1, 3, 5, 6, 7, 8, 12 and 13) had the same gene structures, whereas 4 (TaJAZ4, 9, 10 and 14) had one, two or four exons in each group. In addition, TaJAZ6 and TaJAZ13 lacked intron (Fig. 3). Overall, a highly similar gene structure was exhibited in the duplicated TaJAZ genes.

The TIFY and Jas domains are important for the repressor activity of JAZ proteins in plants. The TIFY and Jas domains locate at the $\mathrm{N}$-terminus and $\mathrm{C}$-terminus of JAZ proteins, respectively. In order to understand the architecture of JAZ proteins in wheat, we scanned these two domains using the MEME web server. As shown in Fig. $1 \mathrm{~b}$ and $\mathrm{c}$, single TIFY and Jas motifs were distributed in each TaJAZ protein and all TaJAZ proteins had TIFY motif at $\mathrm{N}$-terminus while Jas motif at $\mathrm{C}$-terminus. The 


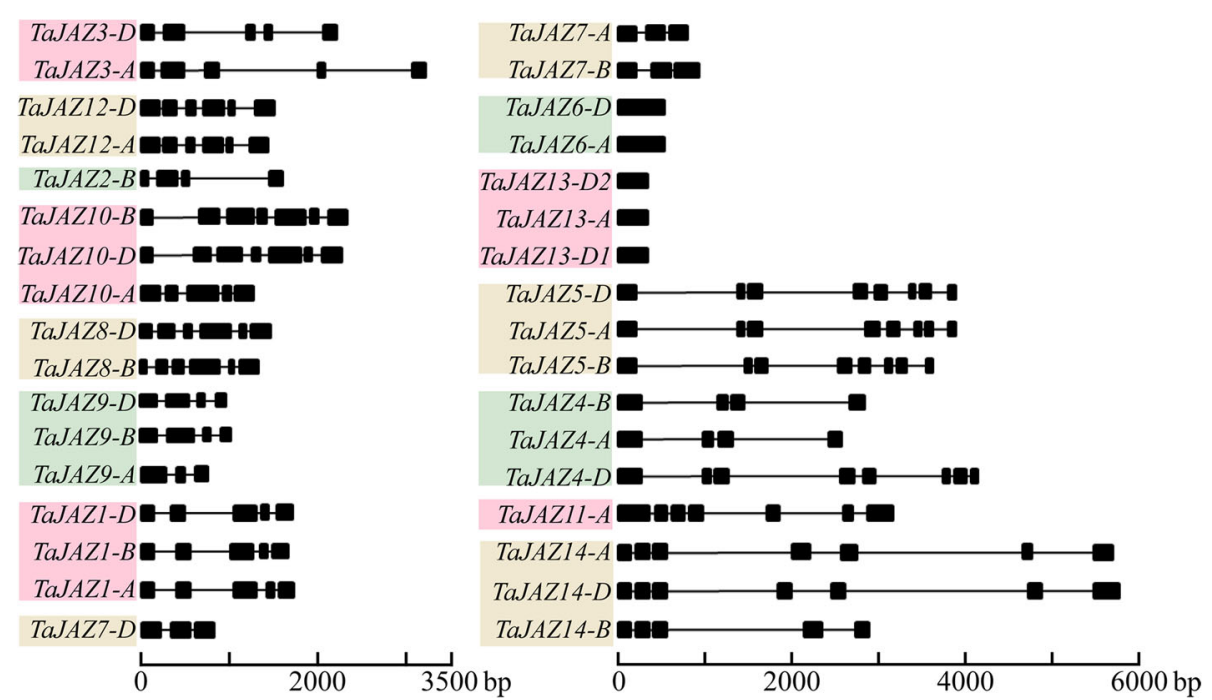

Fig. 3 Exon-intron structures of TaJAZ genes. Exons are represented by blank boxes and introns by blank lines. The sizes of exons and introns could be estimated using the scale below

results were in accordance with the previous studies $[43$, $44]$, indicating that the structures of JAZ proteins were conserved. Moreover, the multi-sequence alignments of protein sequences revealed that the amino acid sequence "TIFY" was changed into "TVFY" in TaJAZ3, "TMFY" in TaJAZ8, "TLFY" in TaJAZ4, "TLVY" in TaJAZ5 and "TLSF" in TaJAZ14 (Additional file 1: Figure S1). The same variations also existed in rice [24], maize [44], and B. distachyon [45]. Besides TIFY domain, the Jas domain is also conserved. In Arabidopsis, Jas domain contains 12-29 amino acids, and the sequences are basically coincident or conserved [46]. But in wheat, the Jas domain in some TaJAZ proteins, such as TaJAZ4, 5 and 14, was inserted into a nuclear localization signal (NLS) sequence (Additional file 1: Figure S1).

\section{Phylogenetic analysis of TaJAZ proteins}

In order to understand the phylogeny of JAZ proteins, $\mathrm{N}-\mathrm{J}$ tree was built using MEGA 6.0 software and the reliability was tested by bootstrap analysis for 1000 replicates. As shown in Fig. 4, all the JAZ proteins derived from different plants were clustered into sub-groups G1G11, and the TaJAZ proteins were clustered into subgroups G3-G11. Sub-group G3 had the maximal number of TaJAZ proteins, and 4 TaJAZ proteins (TaJAZ4, 5, 11 and 14) were clustered into this sub-group. Based on the order that the JAZ proteins appeared in plants, those 11 sub-groups were divided into groups I (marked by red) and II (marked by blue) (Fig. 4). Group I included subgroups G1, G2, G3, G5, G8 and G9, and group II comprised sub-groups G4, G6, G7, G10 and G11. Moreover, we found that the TaJAZ proteins were clustered into the same clades with some AetJAZ, BdJAZ or OsJAZ proteins.
For example, TaJAZ6 was clustered into the sub-group G10 with OsJAZ10, TaJAZ12 was clustered into the subgroup G8 with BdJAZ15, and TaJAZ9 was clustered into the sub-group G6 with AetJAZ2 (Fig. 4). These revealed that the TaJAZ proteins shared a high similarity with those in Ae. tauschii, B. distachyon and rice.

\section{Adaptive evolution analysis of the $T a J A Z$ gene family}

To investigate which type of Darwinian selection determined the process of TaJAZ gene divergence after duplication, the $\mathrm{Ka} / \mathrm{Ks}$ substitution ratio was utilized to the coding sequences of 9 pairs of orthologs between wheat and $B$. distachyon JAZ gene family (Additional file 1: Figure S2). According to the previous research, $\mathrm{Ka} / \mathrm{Ks}$ ratio $<1$ means purifying selection, ratio $=1$ means neutral evolution and ratio $>1$ means positive selection [47]. As shown in Table 2, the $\mathrm{Ka} / \mathrm{Ks}$ ratios of TaJAZ genes ranged from 0.0016 to 0.6973 , suggesting that the TaJAZ gene family had undergone purifying selection in wheat.

\section{Putative cis-acting regulatory elements in the promoter region of TaJAZ genes}

To gain the information about the cis-acting regulatory elements of TaJAZ gene family, the putative promoter region sequence of each TaJAZ gene was analyzed. In this study, promoter sequences were available for 13 of 14 TaJAZ genes, while only the promoter of TaJAZ11 was unavailable due to the limited genome data of wheat. Eight types of putative cis-acting regulatory elements were identified in TaJAZ genes, including LTR element involved in low-temperature responsiveness, TGA element related to Auxin-responsiveness, CGTCAmotif involved in MeJA responsiveness, ABRE associated 


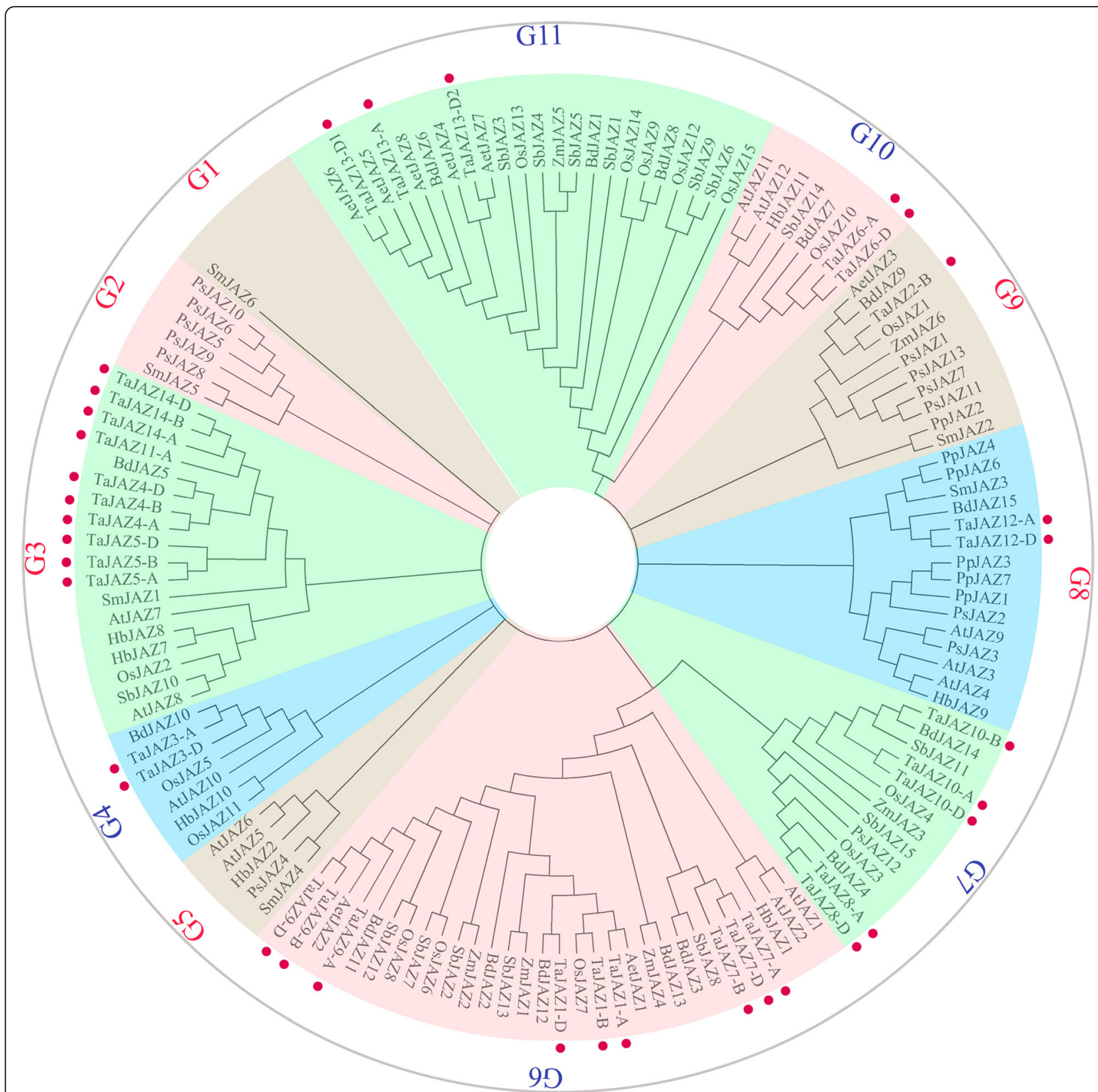

Fig. 4 Phylogenetic relationship of JAZ proteins among wheat and other species. The full-length amino acid sequences of $34 \mathrm{~T}$. aestivum, $6 \mathrm{~S}$. moellendorffii, 12 A. thaliana, 15 O. sativa, 7 P. patens, 6 maize, 15 B. distachyon, 15 S. bicolor, 13 P. sitchensis, 8 Ae. tauschii and 7 H. brasiliensis genes were aligned by using ClustalX and the phylogenetic tree was constructed using MEGA 6.0 by the neighbor-joining method with 1000 bootstrap replicates. Each TaJAZ protein is indicated by a red dot. Two major groups, group I and II, are represented by the red and blue, respectively

with ABA signaling pathway, box-w1 for the response of fungal elicitor, GARE-motif of a gibberellin responsive element regulating gibberellin responsive genes, MBS, a MYB-binding sequence involved in regulation of drought-inducible genes and TCA element regulating the SA related genes (Additional file 2: Table S3). The type and number of cis-acting regulatory elements in the promoter region of each TaJAZ gene were discrepant (Additional file 2: Table S3). These showed that the members of TaJAZ gene family might be able to respond to different abiotic stresses.

\section{Expression profiling of TaJAZ genes under abiotic stresses in TGMS wheat line}

In order to obtain the expression profiling of $\mathrm{TaJAZ}$ genes under plant hormones, low temperature $\left(10{ }^{\circ} \mathrm{C}\right)$, high salinity and drought treatments, qRT-PCR was performed to gain the relative expression pattern of each 
Table $2 \mathrm{Ka} / \mathrm{Ks}$ ratio of the duplicated JAZ genes in wheat using Brachypodium distachyon as an outgroup

\begin{tabular}{llll}
\hline Gene & A-genome & B-genome & D-genome \\
\hline TaJAZ-1 & 0.232 & 0.2405 & 0.2787 \\
TaJAZ-2 & - & 0.3099 & - \\
TaJAZ-3 & 0.3751 & - & 0.3623 \\
TaJAZ-6 & 0.0016 & - & 0.0286 \\
TaJAZ-7 & 0.2753 & 0.3521 & 0.401 \\
TaJAZ-8 & 0.5066 & - & 0.6973 \\
TaJAZ-9 & 0.2887 & 0.2922 & 0.2746 \\
TaJAZ-10 & 0.3754 & 0.3751 & 0.3785 \\
TaJAZ-13 & 0.1222 & - & 0.2213 \\
\hline
\end{tabular}

TaJAZ gene using the 14-day-old TGMS wheat seedlings. As shown in Fig. 5, the transcript profiling of TaJAZ genes showed up-regulation of TaJAZ 1, 2, 3, 4, $7,8,9,10,11$ and 12 and down-regulation for TaJAZ5, 6,13 and 14 in response to MeJA treatment. ABA treatment influenced the expression dynamics of TaJAZ genes. Except that TaJAZ6, 8 and 11 were inhibited, the expression levels of the remaining $\mathrm{Ta} J A Z$ genes were upregulated under ABA treatment (Fig. 5). Under GA treatment, the transcriptional levels of TaJAZ1 and 9 were increased, and TaJAZ6 and 7 were inhibited. The expression patterns of other TaJAZ genes were similar, i.e., up- at 2-4 h or 2-8 h followed by down-regulated at $24 \mathrm{~h}$ (Fig. 5). Under IAA treatment, the expression levels of TaJAZ1, 2 and 10 were up-regulated at time points $2 \mathrm{~h}, 4 \mathrm{~h}$ and $8 \mathrm{~h}$; TaJAZ7 and 9 were rapidly upregulated at $2 \mathrm{~h}$, but down-regulated subsequently; TaJAZ5 was induced at each time point, but inhibited at $4 \mathrm{~h}$. The expression levels of remaining $\mathrm{TaJAZ}$ genes were inhibited in different degree (Fig. 5). Moreover, the expression analysis of TaJAZ genes showed that TaJAZ1, $2,3,7,8$ and 9 were up-regulated under SA treatment, and the rest of TaJAZ genes were down-regulated (Fig. 5). These revealed that all TaJAZ genes could respond to various plant hormones, and they might be involved in some complex signaling pathways.

Under high salinity treatment, the expression levels of TaJAZ1, 2, 5 and 14 were up-regulated, while TaJAZ9 and 10 were up-regulated at $2-4 \mathrm{~h}$ post treatment, but down-regulated at $8 \mathrm{~h}$ subsequently (Fig. 5). The expression levels of TaJAZ12 were reduced at 2-8 h, and increased at $12-24 \mathrm{~h}$. For drought stress, the expression analysis of TaJAZ genes showed that 6 genes (TaJAZ1, 2, $3,9,10$ and 11 ) were up-regulated at $2-8 \mathrm{~h}$ or $2-12 \mathrm{~h}$, whereas TaJAZ6, 8 and 14 were inhibited at each time point after treatment, and TaJAZ4, 5 and 7 were decreased firstly but increased subsequently (Fig. 5). In addition, except TaJAZ5, 11 and 14, we found that TaJAZ genes could respond to low temperature $\left(10{ }^{\circ} \mathrm{C}\right)$, and the transcriptional levels of the rest of genes were obviously up-regulated. Ulteriorly, we found that the expression levels of TaJAZ1, 2, 4, 7, 8 and 10 were upregulated at each time point (Fig. 5). These results

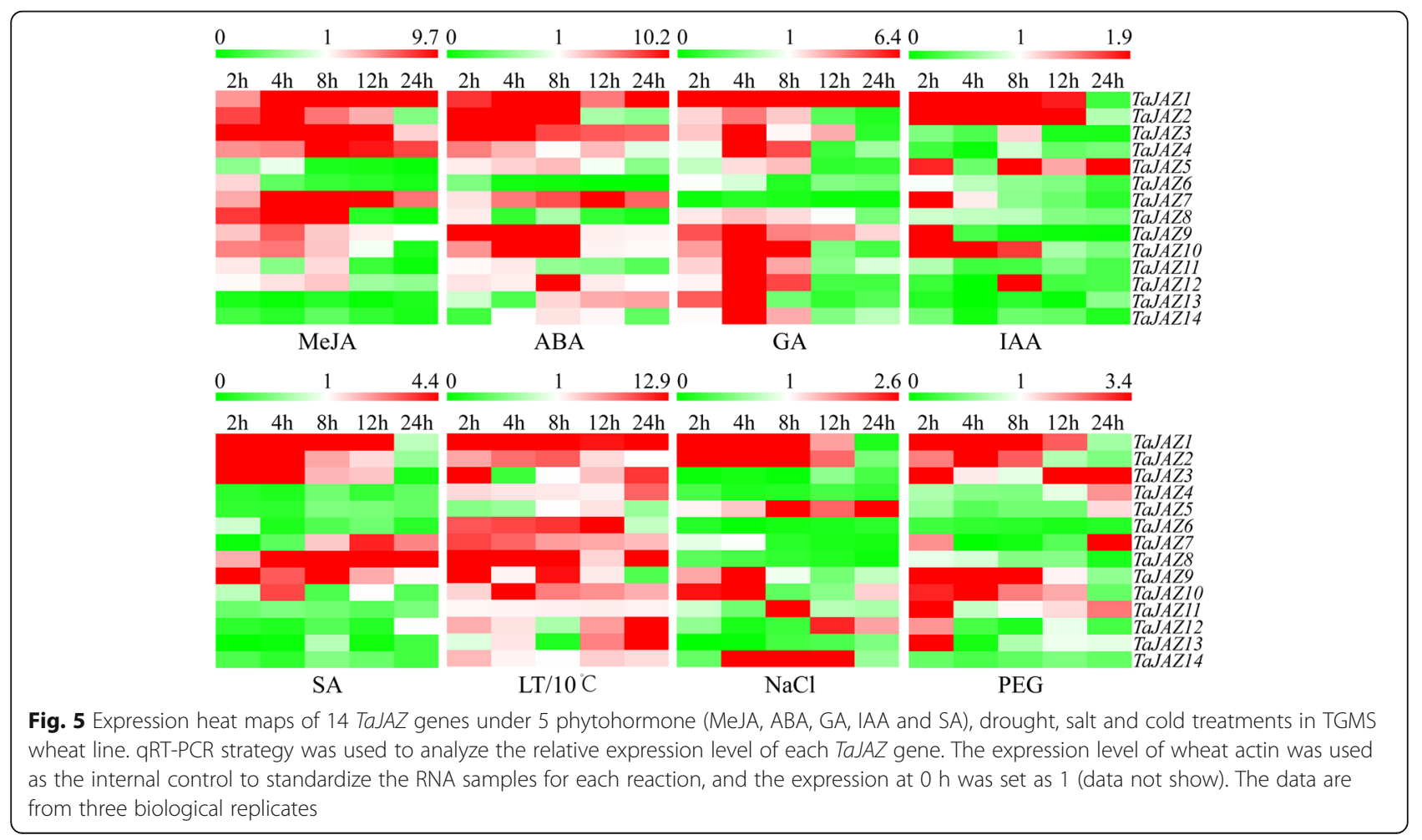


indicated that the members of $T a J A Z$ gene family were highly sensitive to high salinity, drought and low temperature treatments, and might be involved in some very complex regulation networks in TGMS wheat line BS366.

\section{Expression patterns of TaJAZ genes during the whole heading stage}

The TGMS wheat line BS366 exhibits temperaturedependent sterility. The anthers of BS366 are indehiscent in sterile environment (Fuyang, Anhui province, China) [48], and abnormal dehiscence in fertile environment (Beijing, China) (Fig. 6a, c and d). The anthers of the normal control wheat line Jing411 can normally dehisce in fertile environment (Fig. 6a, a and b). In order to explore the relationship between the expression patterns of TaJAZ genes and the abnormal anther dehiscence in TGMS wheat line BS366, the whole heading stage was divided into six periods, from the start of heading to pre-abloom stage (Fig. 6b). qRT-PCR was used to analyze the relative expression levels of each TaJAZ gene in BS366 and Jing411 anther tissues, which were collected from the fertile environment.

During the whole heading stage of BS366 and Jing411, the expression pattern of each $\mathrm{TaJAZ}$ gene was obviously fluctuant and regular (Fig. 6c). Except TaJAZ5, the relative expression levels of the rest of TaJAZ genes were markedly increased at the stage 2 and stage 4 , and the corresponding expression patterns displayed some similarities (Fig. 6c). At the heading stage 6 of BS366, the transcriptional levels of 8 TaJAZ genes (TaJAZ1, 2, 3, 7, 8,1113 and 14) were reduced compared with that in the heading stage 1, whereas the expression levels of TaJAZ4, 5, 6, 9, 10 and 12 were increased (Fig. 6c). At the heading stage of Jing411, the expression levels of TaJAZ1, 2, 3, 11, 12, and 13 were inhibited, but the transcriptional levels of TaJAZ4, 5, 6, 7, 8, 9, 10 and 14 were induced (Fig. 6c). Interestingly, the expression patterns of TaJAZ7, 8 and 12 were discrepant at the heading stage 6 of BS366 and Jing411, but similar at the heading stages $1-5$ (Fig. 6c). These results revealed that the functions of TaJAZ genes were distinctly different, and TaJAZ7, 8 and 12 might play their conclusive roles in regulation of the degree of anther dehiscence at the heading stage 6 in wheat.

\section{Tissue-specific expression profiles of TaJAZ genes}

The qRT-PCR was performed to investigate the tissue expression patterns of TaJAZ genes. The expression profiles of TaJAZ genes were analyzed in different tissues of the TGMS wheat line BS366 and the normal control Jing411 at heading stage 6 (Fig. 6b). As shown in Fig. 7, five TaJAZ genes (TaJAZ1, 4, 10, 11 and 14) were expressed in root, stem, leave, stamen and pistil tissues, and they were expressed constitutively in BS366 and
Jing411. Among the rest of TaJAZ genes, TaJAZ6 and 13 were specifically expressed in root, TaJAZ5, was observably highly expressed in leaf tissues, and TaJAZ2 and 9 were expressed specifically in glume (Fig. 7). The relative expression levels of TaJAZ7, 8 and 12 in stamen tissues were markedly higher than those in other tissues (Fig. 7). In addition, the expression level of TaJAZ3 in root tissues was as high as that in stamen, and none of TaJAZ genes was specifically expressed in stem or pistil tissues (Fig. 7).

\section{Cloning of TaJAZ7-D, 8-D and 12-D and subcellular location}

To investigate the reliability of identified TaJAZ genes, we used the specific primers to clone the ORF sequences of TaJAZ7-D, 8-D and 12-D and confirmed these sequences by DNA sequencing. The results showed that the ORF length and the nucleic acid constituent of TaJAZ7-D, 8-D and $12-D$ were in line with the data that identified from wheat genome database (Additional file 1: Figure S3A). Further, we confirmed the subcellular localization of TaJAZ7-D-GFP, TaJAZ8-D-GFP and TaJAZ12-D-GFP fusion proteins. As expected, all three fusion proteins localized in nucleus in Arabidopsis mesophyll protoplasts cells (Additional file 1: Figure S3B). These revealed that the TaJAZ7-D, 8-D and 12-D were true genes, and directly participated in JA signaling pathway in wheat.

\section{Discussion}

The structural characteristics of JAZ transcription repressors family in bread wheat

To date, the $J A Z$ gene family has been reported in many plants, such as A. thaliana [24] and H. brasiliensis [49], $B$. distachyon [45] and O. sativa [43]. The expression patterns, gene and protein characteristics and the function of some $J A Z$ genes have already been unfolded. However, the relative research on $J A Z$ gene family was still infrequent in wheat. Based on the latest draft of wheat genome data, we isolated a JAZ gene family including 14 members from wheat (Table 1).

In plants, the gene construction of $J A Z$ is manifold, and this multiformity is mainly reflected in the length and number of introns [50]. The length of introns in TaJAZ gene family ranged from 54 to $2098 \mathrm{bp}$. The shortest intron was only $54 \mathrm{bp}$ in TaJAZ7-B, while the longest was 2098 bp in TaJAZ14-D (Fig. 3). Previous study revealed big gaps in the length of introns in $J A Z$ gene family in many plants, ranging from 62 to $4422 \mathrm{bp}$ [50]. We found that the intron length in TaJAZ gene family was in line with this range. In terms of the number of introns, the TaJAZ gene family members had 0-7 introns. TaJAZ5 had the maximum 7 introns, whereas TaJAZ6 and 13 had no introns (Fig. 3). This result was consistent with the number of introns in the $J A Z$ genes 


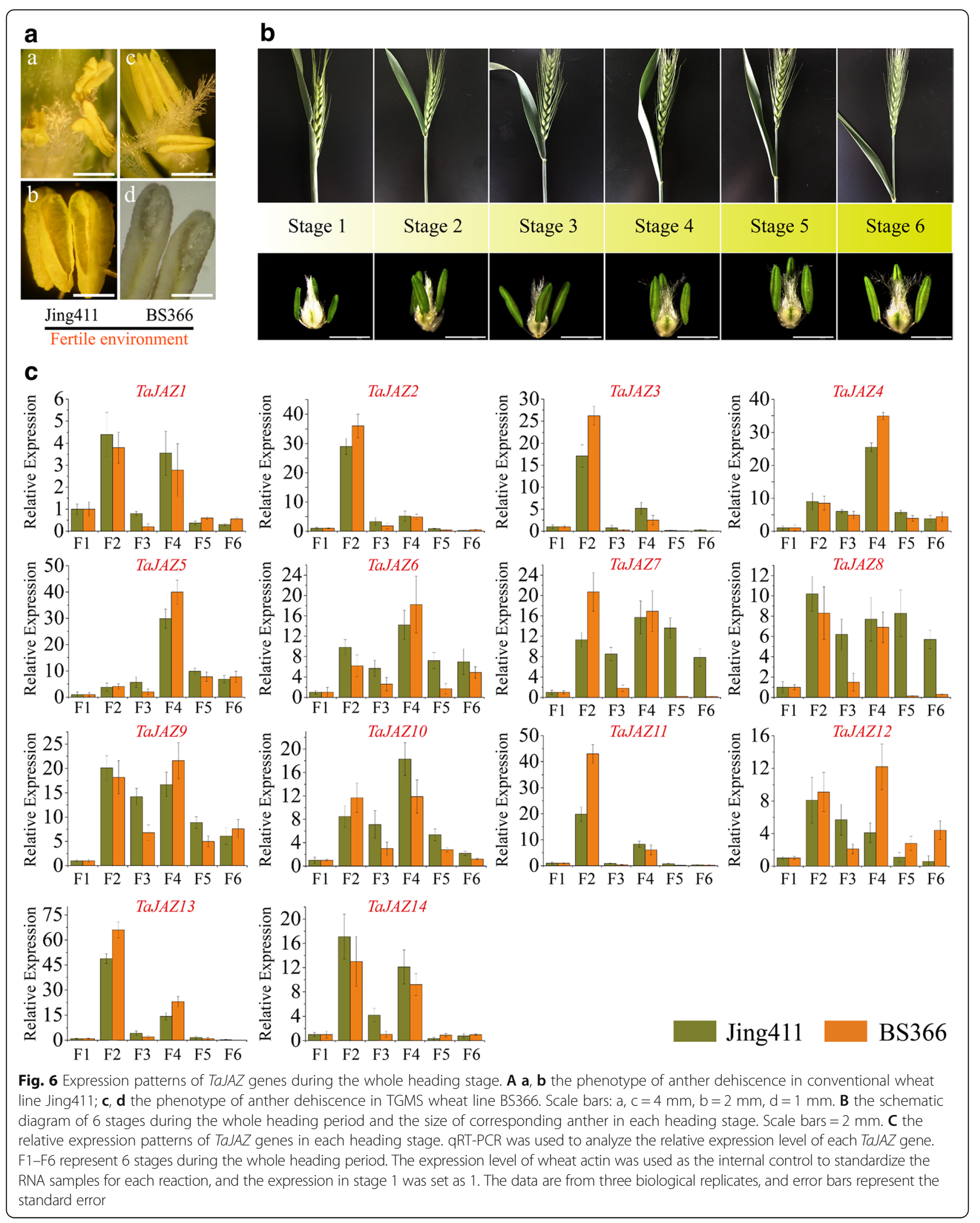




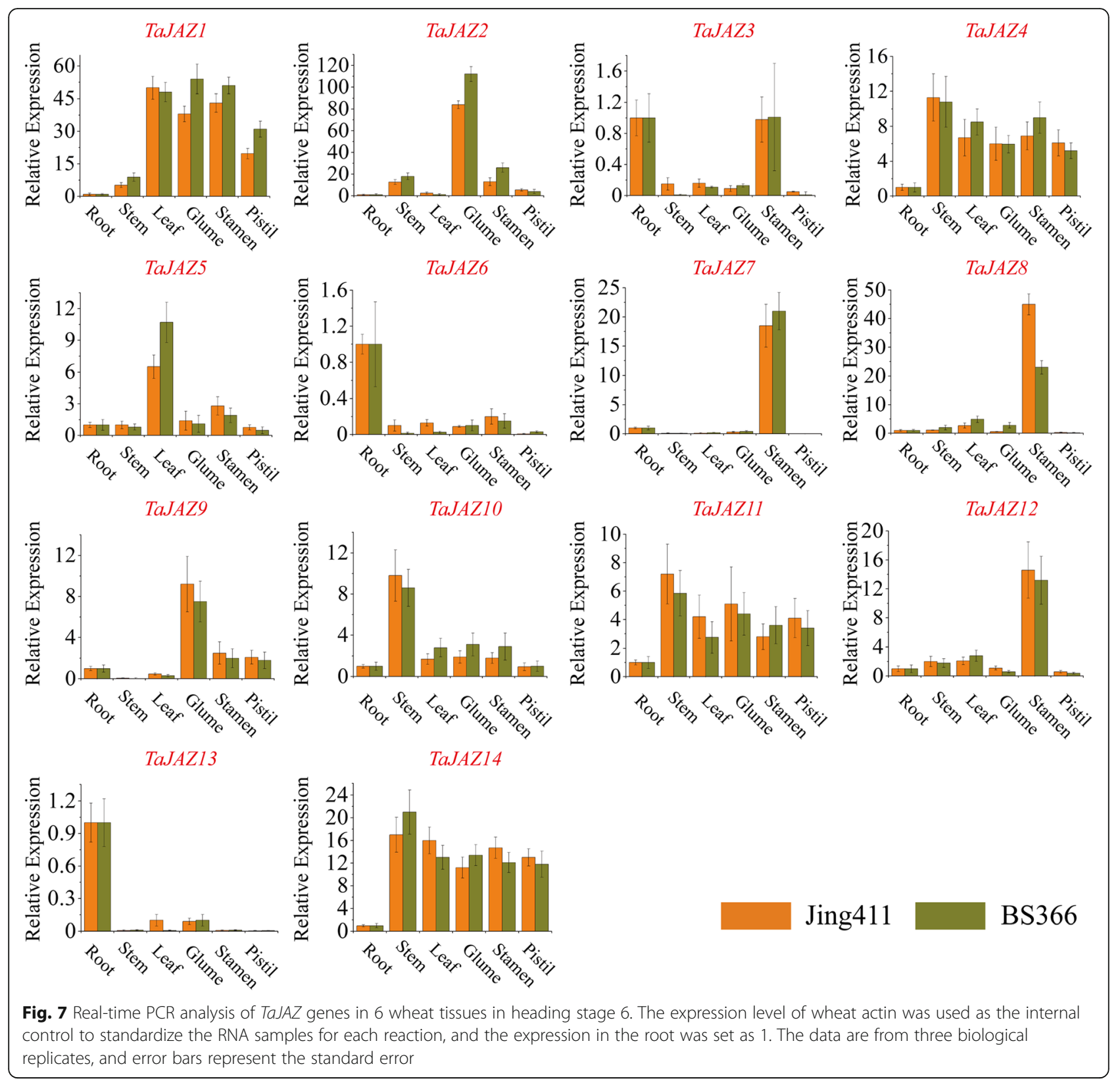

of other plants [50]. However, some discrepancies were found in the number of introns among different copies of the same TaJAZ gene. For example, among the three copies of TaJAZ4, TaJAZ4- $A$ and $-B$ had 3 introns each, whereas TaJAZ4-D had 7 introns. TaJAZ14- $A$ and $-D$ had 6 introns each, whereas TaJAZ14-B had 4 introns. The similar characteristics were also found in the copies of TaJAZ9 and 10 (Fig. 3). This characteristic of gene structure was proper in wheat, because wheat has sub-genomes A, B and D. Overall, the results mentioned above suggested that the phenomenon of intron indels or intron lose occurred in TaJAZ genes during the long evolutionary process, resulting in various structures of TaJAZ genes.
Generally, a typical JAZ protein has one TIFY and one Jas domain at its $\mathrm{N}$ - and C-terminal, respectively. TIFY domain has two main functions, with one as a medium for the interaction of homomeric and heteromeric complexes formation, and the other as a medium for the interaction between JAZ proteins and MYC transcription factors [24]. The other distinguishing feature of JAZ proteins is the highly conserved Jas domain located near the C-terminus [30]. It is well known that Jas domain participates in protein-protein interaction with both COI and transcription factors, such as MYC2 [26, 51], and the nuclear localization signal (NLS) is included in this domain sequence [52]. In the present study, all TaJAZ 
proteins contained one TIFY and one Jas domain, but the amino acid composition of TIFY and Jas domain was different. The multiple sequence alignment showed that some amino acid substitutions existed in the core sequence of TIFY domain (Additional file 1: Figure S1). For example, the protein sequence "TIFY" was replaced with "TVFY" in TaJAZ3, "TMFY" in TaJAZ8, and "TLFY" in TaJAZ4. This architectural feature is familiar in JAZ proteins in many other plants [43, 45, 49]. The protein sequences of Jas domain were relatively conserved, and the sequence lengths were almost coincident in Arabidopsis and gramineous rice and maize [24, 43, 44]. But in wheat, the Jas motif sequences of some $J A Z$ proteins, such as TaJAZ4, 5 and 14, were inserted by a short and highly similar NLS-like peptide, respectively (Additional file 1: Figure S1). This structural feature was rare in other plant JAZ protein, and this specific structure was only available in wheat JAZ proteins.

In conclusion, there were some similarities and otherness in the characteristics between TaJAZ gene family and other plant $J A Z$ gene families. The results mentioned above suggested that the $T a J A Z$ gene family might have multiple functions due to the complex structural characteristics as same as $J A Z$ gene families in many other plants.

\section{The putative functions of TaJAZ gene family}

In order to gain more insights into the function of TaJAZ genes, we analyzed the cis-acting regulatory elements composition and the expression patterns under various stresses. Cis-acting regulatory elements are important molecular switches involved in the regulation of gene transcription under abiotic or biotic stresses [53]. In this study, we mainly detected the cis-acting regulatory elements that can respond to plant hormones (ABA, IAA, MeJA, GA and SA) and stress tolerances (low temperature, drought and high salinity). Due to the restriction of genomic sequencing data, we failed to gain the promoter region of TaJAZ11. In wheat JAZ gene promoter regions, the differences were mainly in the number and type of cis-acting regulatory elements. For example, the promoter region of TaJAZ1 had 4 types of cis-acting regulatory elements, i.e. ABRE (the number was 4), TGA (the number was 2), box-w1 (the number was 2) and CGTCA-motif (the number was 1), while TaJAZ6 only had 2 ABRE and 2 MBS elements (Additional file 2: Table S3). The promoter sequences of some TaJAZ genes lacked some types of cis-acting regulatory elements, but the expression patterns revealed that all $T a J A Z$ genes could respond to all 8 treatments. For example, we found that all TaJAZ genes had no high salinity response-related cis-acting regulatory elements, but the relative expression levels of all TaJAZ genes were up- or down-regulated under high salinity treatment
(Fig. 5). This indicated that the gene expression level under different treatments was not only dependent on the presence of relevant cis-acting regulatory elements, but also might be regulated by other physiological pathways in wheat.

To further investigate the biological functions of TaJAZ genes, we used different phytohormone to treat the wheat seedlings. Under MeJA treatment, the relative expression levels of TaJAZ1, 2, 3, 4, 7, 8, 9, 10 and 12 were obviously increased, while TaJAZ5, 6, 11, 13 and 14 were inhibited. By combining the cis-acting regulatory elements analyzed results, we found that the promoter sequences of TaJAZ2, 4 and 6 lacked the MeJAresponsive elements. Thus, we assumed that TaJAZ1, 3, $5,7,8,9,10,12,13$ and 14 were directly involved in JA signaling pathway in wheat, and TaJAZ2, 4 and 6 could also respond to MeJA treatment by other unknown regulative pathways. Likewise, for GA treatment, the relative expression levels of TaJAZ6 and 7 were depressed, and the rest of TaJAZ genes were up-regulated. Based on the composition of cis-acting regulatory elements in promoter sequences, only TaJAZ3, 5, 7 and 12 had GARE element involved in GA response, but the TaJAZ genes lacking GARE element could also respond to GA. For IAA treatment, only TaJAZ5, 7, 8, 12 and 13 had the TGA-element, but all TaJAZ genes could obviously respond to it. For SA treatment, only TaJAZ3, 7, 8, 10 and 13 had the TCA-element, but the response was available in all genes. For ABA treatment only TaJAZ2, 4, 6, 8 and 12 had no ABRE element, but they were still sensitive to the presence of ABA (Fig. 5 and Additional file 2: Table S3). Given this, we found that some TaJAZ genes could respond to various phytohormones, and they might be directly or indirectly involved in the phytohormone crosstalk.

It is clear that the phytohormone crosstalk is universally available in plants, and a very complex signaling regulative network, which constituted by many phytohormone crosstalk, plays a very important role in the regulation of growth and development in plants. There is no doubt that JA signaling pathway plays a leading role in connecting different phytohormone signaling pathways [33]. As a repressor in downstream of JA signaling, JAZ proteins were irreplaceable, and they are the linkers among different crosstalks [33]. By synthesizing the analytic results of the cis-acting regulatory elements composition and the expression patterns under abiotic stresses (Fig. 5 and Additional file 2: Table S3), we speculated that TaJAZ3, 5, 7 and 12 were involved in the JAGA crosstalk, which is a crosstalk to promote plant growth and defense against pathogens. Moreover, the JA-GA crosstalk can also act synergistically during stamen development, and JAZ proteins appear to play a significant role in this developmental function by 
interacting with the transcription factors $M Y B 21$ and MYB24, which both required for JA- and GA-mediated stamen development and male fertility [54]. Therefore, we thought that TaJAZ3, 5, 7 and 12 had a close contact with the development of stamen in wheat. The mechanism of JA-SA crosstalk in plant remains largely unknown, but the previous evidence has shown the effect of SA on JA signaling through direct or indirect regulation of the stabilization of JAZ proteins $[55,56]$. This crosstalk can protect plants from the biotic and abiotic stresses, especially the pathogen infection $[57,58]$. Here, we found that TaJAZ3, 7, 8, 10 and 13 had two types of cis-acting regulatory elements CGTCA-motif and TCAelement, and they were sensitive to JA and SA treatments (Fig. 5 and Additional file 2: Table S3). Thus, we thought that the functions of TaJAZ3, 7, 8, 10 and 13 were to enhance or inhibit the antiviral ability of plants. IAA is a very important phytohormone and plays vital roles during the development of plants [59]. The evidences revealed that the function of JA-IAA crosstalk was mainly embodied in regulating the root meristem activity and stem cell maintenance via antagonistic effect in plants [60-62]. Based on the results of cis-acting regulatory element component and the expression patterns (Fig. 5 and Additional file 2: Table S3), we assumed that TaJAZ5, 7, 8, 12 and 13 were directly participated in the JA-IAA crosstalk, and they might be involved in the regulation of primary root growth in wheat. ABA is a "stress hormone" that can regulate growth, stress tolerance, seed germination and senescence in plants. In JAABA crosstalk, both synergetic and antagonistic interactions are well known, and it is possible that JAZ proteins play important roles in regulating the JA-ABA crosstalk [33]. Given this, except TaJAZ2, 4, 6, 8 and 12, which lacked ABRE element, we speculated the rest of TaJAZ genes took part in the JA-ABA crosstalk, and played a role in plant stress tolerance, such as high salinity, drought and low temperature stresses (Fig. 5 and Additional file 2: Table S3).

In addition, the analysis of phylogenetic relationship among the $J A Z$ genes can also reveal the putative function of them, because the homologous genes usually have similar biological functions [63]. In this study, we found that TaJAZ2 was clustered with OsJAZ1 into the sub-group G9 (Fig. 4). It is clear that OsJAZ1 protein interacts with a basic helix-loop-helix protein, OsbHLH148, to regulate the drought tolerance in rice [64]. Here, we found 4 drought-inducible cis-acting regulatory elements MBS in the promoter sequence of TaJAZ2, and the expression level of TaJAZ2 was also variational under drought stress. Thus, TaJAZ2 was likely participated in drought tolerance in wheat. Then, we found that TaJAZ4 was clustered with BdJAZ5 into the sub-group G3 (Fig. 4). The expression levels of BdJAZ5 are increased under salt, cold and heat treatments in B. distachyon [45]. There were 2 low temperature related cis-acting regulatory elements LTR in the promoter region of TaJAZ4, and the expression pattern of TaJAZ4 was similar with that of BdJAZ5 under cold stress, so TaJAZ4 might be involved in cold response in wheat. Further, we found that TaJAZ6 shared a high similarity with BdJAZ7 in sub-group G10 (Fig. 4), and there were $2 \mathrm{MBS}$ elements in its promoter sequence. The expression patterns of TaJAZ6 and BdJAZ7 were similar under drought stress (Fig. 5) [45], indicating that TaJAZ6 was also involved in drought tolerance in wheat. Overall, the functions of many TaJAZ genes were overlapping, which needed to be studied minutely in the future.

\section{The evolution analysis of JAZ transcription repressors family}

In the ancient terricolous plants, there are 7 and 6 members in the JAZ gene family in P. patens and S. moellendorffii genome, respectively [50]. In neonatal terricolous plants, there are $13 J A Z$ genes in gymnospermous $P$. stichensis genome, $12 \mathrm{JAZ}$ genes in dicotyledonous $A$. thaliana genome, 15, 16 and $15 J A Z$ genes in gramineous $B$. distachyon, $S$. bicolor, and $O$. sativa genomes, respectively [50]. In the present study, 14 TaJAZ genes were identified from wheat genome (Table 1), and this number was similar to those in gramineous plants, but obviously more than those in older plants $P$. patens and $S$. moellendorffii. This result suggested that the number of $J A Z$ genes in higher plants undergone the expansion, and became stable subsequently. Based on the analysis of chromosome localization, we found that the chromosome distribution of TaJAZ genes was tufted. For example, the copies of TaJAZ4, 5, 6 and 7 were densely distributed on the chromosomes $4 \mathrm{~A}, 4 \mathrm{~B}$ and $4 \mathrm{D}$, respectively (Fig. 2). In addition, both TaJAZ4 and 5 were clustered into sub-group G3 (Fig. 4), while TaJAZ6 and 7 were clustered into sub-groups G10 and G6 (Fig. 4), respectively, indicating that TaJAZ4 and 5 undergone the event of tandem duplication, and maybe the event of divergence happened in the evolutionary process of TaJAZ genes. Moreover, we found that a pair of tandem TaJAZ genes, TaJAZ13-D1 and $-D 2$, shared a high similarity in their protein sequences (Additional file 1: Figure S1), exhibiting that the duplication event of these two genes also happened. Given this, we thought that the gene tandem duplication was the main result leading to the augmentation in the number of TaJAZ gene.

Based on the N-J phylogenetic tree, all JAZ proteins from different plants were clustered into 11 subgroups (Fig. 4). The sub-groups G1, G2, G3, G5, G8 and G9 each had one SmJAZ protein, while none was present in the rest of sub-groups. Thus, we separated these 11 subgroups into groups I (marked by red) and II (marked by blue) (Fig. 4). In group I, the sub-group G1 had only one 
JAZ protein, SmJAZ6, indicating that all JAZ proteins in terrestrial plants originated from a common ancestor. In sub-groups G3 and G5, the JAZ proteins from S.moellendorffii and other plants, such as $T$. aestivum, $B$. distachyon and $A$. thaliana, were clustered in these two clades, indicating that the differentiation of these JAZ proteins might have predated the divergence between flowering plants and pteridophyte (Fig. 4). In sub-groups G8 and G9, the JAZ proteins from $P$. patens and other plants, such as $A$. thaliana, and $O$. sativa, were clustered in these two sub-groups, suggesting that the differentiation of those JAZ proteins might have predated the divergence between bryophyte and tracheophyte (Fig. 4). In group II, the JAZ proteins from gymnospermous $P$. sitchensis and other angiosperm were included in G7, suggesting that those JAZ proteins might have predated the divergence between gymnosperms and angiosperms (Fig. 4). In addition, sub-groups G4, G6 and G10 comprised some JAZ proteins from monogenus and dicotyledonous plants, indicating that these JAZ proteins appeared before the divergence between monogenus and dicotyledonous plants (Fig. 4). The sub-group G11 only included the JAZ proteins from gramineous plants (Fig. 4), suggesting that the differentiation of these JAZ proteins ahead of the formation of gramineous plants.

For TaJAZ protein family, we found that all TaJAZ proteins were directly clustered with the JAZ proteins from Ae. tauschii, B. distachyon, S. bicolor or O. sativa. For example, TaJAZ3 was clustered with BdJAZ10 into sub-group G4; TaJAZ9 was clustered with AetJAZ2 into G6; TaJAZ10 was clustered with BdJAZ14 and SbJAZ11 into G7; and TaJAZ13 was clustered with BdJAZ6 and AetJAZ4, 5, 6, 7 and 8 (Fig. 4). This could be attributed to the fact that these 5 plant species are gramineous. Thus, we speculated that the differentiation of TaJAZ protein family occurred after the divergence between monocotyledon and dicotyledon (90 MYA), and ahead of the formation of gramineous plants (50-80 MYA). In addition, the $\mathrm{Ka} / \mathrm{Ks}$ ratio revealed that the TaJAZ protein family undergone a process of purifying selection (Table 2), suggesting that the TaJAZ protein family tended to be stable during the long evolutionary process.

\section{TaJAZ7, 8 and 12 were involved in the abnormal anther dehiscence}

It is clear that JA, as a kind of important phytohormone, is widely involved in the regulation of anther dehiscence, filaments elongation and pollen fertility in plants [65]. The JA biosynthesis and signaling pathways were important for the development of anther during late developmental stage [66]. JAZ proteins, as repressors in JA signaling pathway, inhibit the transcription of JA response genes [33], and there is no doubt that JAZ genes play an essential role in the JA-mediate regulation pathway of anther dehiscence in plants [66]. The TGMS wheat line BS366 is a temperature dependent variety and its fertility can convert under different environments [48]. Hybrid seed could be produced under sterile condition (TGMS line BS366 as maternal plant), while TGMS line itself could be propagated under fertile condition [41]. The anther dehiscence of conventional wheat line Jing411 is normal under fertile condition (Fig. 6a, a and b), and the pollen can spill out from anthers smoothly. In sterile environment, the anther of TGMS wheat line BS366 is absolutely indehiscent [48], resulting in the male sterility. Interestingly, we found that the anther of BS366 could not fully dehisce, and only the topmost part of anther could crack (Fig. 6a, c and d), leading to the pollen spilling out incompletely. This abnormal phenotype was profitless for the seed multiplication of TGMS wheat line BS366.

In order to explore the potential relationship between the expression patterns of TaJAZ genes and the phenomenon of abnormal anther dehiscence, we divided the heading stage into six periods, and the expression pattern of each TaJAZ gene was checked in the anther tissues of BS366 and Jing411.. As shown in Fig. 6c, the expression patterns of all $T a J A Z$ genes were highly similar from stage 1 to stage 5 , and the relative expression levels in stage 2 and stage 4 increased obviously. Given this, we speculated that all $14 \mathrm{TaJAZ}$ genes played important roles in regulation of anther development at stages 1, 2, 3, 4 and 5. Further, we noticed that the relative expression levels of TaJAZ genes at stage 6 were distinguishing in the anther of BS366, and the expression patterns were mainly divided into two types. The first category included TaJAZ1, 2, 3, 7, $8,11,13$ and 14 , and the expression levels of these genes were inhibited (Fig. 6c). The second category included TaJAZ4, 5, 6, 9, 10 and 12, and the expression levels of these genes were induced (Fig. 6c). In the anther of Jing411, the expression patterns of TaJAZ1, 2, 3, 4, 5, 6, 9, $10,11,13$ and 14 were as same as those in BS366 at the heading stage 6 (Fig. 6c). It was obvious that the expression levels of TaJAZ7, 8 and 12 in the anther of Jing411 were adverse to those in BS366 at stage 6. For example, in the anther of BS366, the expression level of TaJAZ7 was inhibited at stage 6, exhibiting 18 times lower than that at stage 1 (Fig. 6c). In contrast, the transcriptional level of TaJAZ7 increased at the heading stage 6 in the anther of Jing411, displaying 7 to 8 folds higher than that at stage 1 (Fig. 6c). Similar results were also found in the expression patterns of TaJAZ8 and 12. These indicated that not all TaJAZ genes were involved in the regulation of anther dehiscence, and the genes with the same expression patterns in the anthers of Jing411 and BS366 may not be involved in the regulation of the anther dehiscence.

Moreover, the tissue-specific expression assay was performed to check the expression levels of TaJAZ genes in different tissues of BS366 and Jing411.. The relative 
expression levels of TaJAZ1, 2, 4, 10, 11 and 14 were obviously high in stamen tissues, but they all had high expression levels in other tissues (Fig. 7). Therefore, we thought that the regulatory effect of these genes was constitutive. The relative expression levels of TaJAZ7, 8 and 12 were markedly higher in stamen than that in other tissues, showing a high degree of expression specificity. For most of TaJAZ genes, the tissue-specific expression patterns were consistent in the anther tissues of Jing411 and BS366. In addition, the subcellular localization showed that TaJAZ7, 8 and 12 were all located in nucleus (Additional file 1: Figure S3). Based on the results mentioned above, we thought that TaJAZ7, 8 and 12 were directly participated in JA signaling pathway, and most likely to directly regulate the abnormal anther dehiscence. Thus, TaJAZ7, 8 and 12 were regarded as the candidate genes for the regulation of abnormal anther dehiscence in TGMS wheat line. The functions of TaJAZ7, 8 and 12 will be analyzed in our future works.

\section{Conclusions}

Fourteen $J A Z$ family genes were identified from common wheat genome. The structure analysis revealed that TaJAZ gene family had unique characteristic in protein structure. JAZ proteins from wheat and some other plants were classified into 11 different orthologous groups, showing that all JAZ proteins from terrestrial plants derived from the same ancestor, and TaJAZ proteins shared high similarity with the JAZ proteins from Ae. tauschii, $B$. distachyon, and $O$. sativa. The $\mathrm{Ka} / \mathrm{Ks}$ ratio of TaJAZ family genes were very low (less than 1), suggesting that these genes had been under purifying selection in the evolutionary histories.

Moreover, we found that wheat $J A Z$ genes showed differential tissue-specific expression patterns responsive to abiotic stresses. TaJAZ7, 8 and 12 directly participated in JA signaling pathway, and closely involved in the regulation of the abnormal anther dehiscence in TGMS wheat line. In conclusion, these results enriched our knowledge of JAZ gene family in plants, and provided novel candidate genes for improving the TGMS wheat line in seed reproduction.

\section{Methods}

\section{The sources of sequence data}

The whole-genome sequences of Triticum aestivum ( $T$. aestivum, Ta) was downloaded from the wheat genome URGI database (http://wheat-urgi.versailles.inra.frl) and Ensembl database (http://plants.ensembl.org). The JAZ protein sequences of Physcomitrella patens (P. patens, $\mathrm{Pp})$, Selaginella moellendorffii (S. moellendorffii, Sm), Sorghum bicolor (S. bicolor, Sb), Brachypodium distachyon (B. distachyon, $\mathrm{Bd}$ ), and Zea mays (Z. mays, $\mathrm{Zm}$ ) were obtained from JGI database (http://genome.jgipsf.org/). The JAZ protein sequences of Picea sitchensis ( $P$. sithensis, Ps) were achieved from NCBI database (http:// www.ncbi.nlm.nih.gov/). The JAZ protein sequences of Arabidopsis thaliana (A. thaliana, At) were acquired from TIAR database (http://www.arabidopsis.org/). The JAZ protein sequences of Oryza sativa (O. sativa, Os) were collected from TIGR database (http://www.tigr.org/ tdb/ezkl/). The JAZ protein sequences of Hevea brasiliensis $(H$. brasiliensis, $\mathrm{Hb}$ ) were isolated following Hong et al. [49]. The JAZ protein sequences of Aegliops tauschii (Ae. tauschii, Aet) were isolated according to our previous study [67]. All information of sequences was listed in Additional file 2: Table S2.

\section{Identification of $J A Z$ gene family members in wheat}

Based on the wheat genome sequences, a local nucleotide and protein database was established by NCBI local BLAST program (Ftp://ftp.ncbi.nlm.nih.gov/blast/executables/blast+/LATEST/). The hidden Markov model (HMM) profiles PF06200 (TIFY domain) and PF09425 (Jas domain) of the $J A Z$ family were extracted from the Pfam database (http://pfam.sanger.ac.uk) and these two HMM profiles were used to search the local wheat protein database for target hits with the TIFY domain and Jas domain by HMMER 3.0 (http://hmmer.janelia.org/). All non-redundant sequences with E-values lower than 1.0E-05 were selected and received a conserved domain check using the SMART web server (http://smart.emblheidelberg.del) and Pfam tool (http://pfam.xfam.org/). Then, the coding sequences and genome sequences of TaJAZ genes were extracted from the local nucleotide database using the sequence ID of TaJAZ proteins.

\section{Analysis of gene structures, protein motifs and cis-acting} regulatory elements

To illustrate the structures of TaJAZ genes, the coding sequence of each $J A Z$ gene was aligned with its genomic sequence using the Gene Structure Display Server (GSDS) program (http://gsds.cbi.pku.cn/index-php). Multiple Ex prectation Maximization for Motif Elicitation (MEME) was used to identify the motifs of TaJAZ proteins with default settings. To analyze the putative cis-acting elements in a promoter region, $1.5 \mathrm{~kb}$ region upstream of the start codon in each TaJAZ gene was scanned in the PlantCARE database (http://bioinformatics.psb.ugent.be/ webtools/plantcare/html/).

\section{Phylogenetic relationship, chromosome distribution and naming convention of TaJAZ genes}

In order to understand the phylogenetic relationship of TaJAZ genes, the unrooted phylogenetic tree was built using MEGA 6.0 via the Neighbor Joining (NJ) method. The position of each $J A Z$ gene in the corresponding 
chromosome was confirmed by BLAST, and the alignment results were displayed using the MapInspect software (http:// mapinspect.software.informer.com/). Duplicated genes in the branch ends of each group belonging to the A, B or D sub-genomes of wheat were considered as the homologous copies of the same JAZ gene. All TaJAZ genes were named according to their chromosome position and homology among the three wheat sub-genomes [68].

\section{Multiple sequence alignment and phylogenetic analysis of TaJAZ proteins}

Multiple sequence alignment of the candidate TaJAZ proteins was performed using the Clustal X software (Version 1.81 ) with default settings. Meanwhile, $6 \mathrm{SmJAZ}, 12$ AtJAZ, 15 OsJAZ, 7 PpJAZ, 6 ZmJAZ, 15 BdJAZ, 15 SbJAZ, 13 PsJAZ, 8 AetJAZ and $7 \mathrm{HbJAZ}$ proteins were included in the phylogenetic analyses. The unrooted tree was performed using the NJ method of MEGA 6.0 software with bootstrap values from 1000 replicates.

\section{Ka and Ks calculations}

The Ka and Ks calculation method was described previously [68]. Briefly, the orthologous $J A Z$ gene pairs between wheat and $B$. distachyon were used to calculate $\mathrm{Ka}$ and $\mathrm{Ks}$ in the PAL2NAL server using the codeml program of phylogenetic analysis by maximum likelihood [69]. The B. distachyon was used as an outgroup.

\section{Plant materials, treatments and sample collections}

The thermo-sensitive genic male sterile (TGMS) wheat line BS366 and normal wheat line Jing411 were planted in the experimental fields in Beijing (China, N 39 $54^{\prime}, \mathrm{E}$ $\left.116^{\circ} 18^{\prime}\right)$, and managed conventionally. For expression pattern analysis of TaJAZ genes in Jing411 and BS366, the anther tissues were sampled at six stages during the whole heading periods (Fig. 6b).

For tissue-specific expression analyses, 6 tissues (root, stem, leaf, glume, stamen and pistil) from Jing411 and BS366 were collected at the heading stage 6 (Fig. 6b). For abiotic stresses, BS366 seeds were germinated at $25^{\circ}$ $\mathrm{C}$, then the seedlings were cultured in green house for $16 \mathrm{~h}$ light $/ 8 \mathrm{~h}$ dark at $25{ }^{\circ} \mathrm{C}$. After 2 weeks, the seedlings were sprayed with $2 \mathrm{mM} \mathrm{SA}, 100 \mathrm{mM}$ MeJA, $100 \mathrm{mM}$ GA3, $50 \mathrm{mM}$ IAA, and $100 \mathrm{mM}$ ABA that were dissolved in $0.1 \%(v / v)$ ethanol. The control plants were treated with $0.1 \%(v / v)$ ethanol. The leaf tissues from seedlings were collected at $0,2,4,8,12$ and $24 \mathrm{~h}$ post treatment. For the high-salinity and drought treatments, the roots of wheat seedlings were soaked in $200 \mathrm{mM}$ $\mathrm{NaCl}$ and PEG6000 (-0.5 MPa), respectively. The leaf tissues from seedlings were sampled at $0,2,4,8,12$ and $24 \mathrm{~h}$ post treatment. For low temperature stress, the 2week-old seedlings were moved to the incubator in which the temperature was set at $10{ }^{\circ} \mathrm{C}$. The leaf tissues were collected at $0,2,4,8,12$ and 24 h post treatment. All the samples mentioned above were rapidly frozen in liquid nitrogen and stored at $-80{ }^{\circ} \mathrm{C}$ freezer for RNA extraction.

For subcellular localization, Arabidopsis ecotype Col-0 was used for protoplast preparation. Arabidopsis seedlings were planted in greenhouse with a $16 \mathrm{~h}$ light $/ 8 \mathrm{~h}$ dark and a $22 / 20{ }^{\circ} \mathrm{C}$ day/night temperature cycle.

\section{Total RNA extraction and qRT-PCR}

For the expression analysis of TaJAZ genes, total RNA was isolated from wheat tissues using TRIzol reagent (Invitrogen, USA) according to the manufacturer's instructions. First-strand cDNA synthesis was performed using a PrimeScript ${ }^{\mathrm{TM}}$ RT Reagent Kit with gDNA Eraser (TaKaRa, Japan). qRT-PCR was carried out using an ECO Real-time PCR system (Illumina, USA) with SYBR $^{\oplus}$ Permix Ex Taq ${ }^{\mathrm{TM}}$ (TaKaRa, Japan). Primer premier 5.0 program was used to design the primers. Wheat Actin gene (GenBank accession: AB181991) was used as the reference control.

The qRT-PCR for each assay was set up as a $10-\mu \mathrm{L}$ reaction mixture containing $1.0 \mu \mathrm{L}$ of $\mathrm{cDNA}, 5 \mu \mathrm{L}$ of fluorescent reagent SYBR, $3 \mu \mathrm{L}$ of $\mathrm{ddH}_{2} \mathrm{O}$, and $0.5 \mu \mathrm{L}$ each of forward and reverse primers. The reactions were as follows: $95{ }^{\circ} \mathrm{C}$ for $30 \mathrm{~s}, 45$ cycles of $95{ }^{\circ} \mathrm{C}$ for $5 \mathrm{~s}$ and $58{ }^{\circ} \mathrm{C}$ for $30 \mathrm{~s}$. For the melting curve analysis, a program of $95{ }^{\circ} \mathrm{C}$ for $15 \mathrm{~s}$ followed by a constant increase from $55{ }^{\circ} \mathrm{C}$ to $95{ }^{\circ} \mathrm{C}$ was included after the PCR cycles. The expression analysis of TaJAZ genes and reference gene was performed using the same PCR program as detailed above or with a slightly adjusted annealing temperature. The relative expression levels of TaJAZ genes were detected using the comparative threshold cycle method 2 $-\Delta \Delta C \mathrm{C}$ [70]. The primers are listed in Additional file 2: Table S1, and the analysis was confirmed in triplicate.

\section{Cloning of TaJAZ7-D, TaJAZ8-D and TaJAZ12-D, subcellular localization and morphological observation}

The nucleotide sequences of TaJAZ7-D, 8-D and 12-D, retrieved from local wheat nucleotide database, were used for designing specific primers to amplify the corresponding ORF sequences. Then, the ORF sequences of the three genes were inserted into pMD18-T vector, and confirmed by DNA sequencing. Next, the three ORF sequences (no termination codon) with restriction enzyme sites were amplified by PCR strategy and the products were digested using corresponding enzymes. Finally, the ORF sequences of the three TaJAZ genes were inserted into the 16318hGFP (p35S::GFP) vector and produced the vectors p16318-TaJAZ7-D, p16318-TaJAZ8-D and p16318-TaJAZ12-D, respectively. Arabidopsis mesophyll protoplasts cells were isolated according to Yoo et al. [71]. The three fusion plasmids or control vector 
16318hGFP were transformed into Arabidopsis mesophyll protoplasts cells using the PEG4000-mediated method [72]. GFP signal was detected by laser confocal fluorescence microscopy (ZEISS LSM 880, Germany). The representative spikelets and anthers were imaged using a Leica MZ16F stereomicroscope (Leica Microsystems, Wetzlar, Germany). The spikes of wheat seedlings were imaged using a digital SLR camera (Canon, EOS 700D).

\section{Additional files}

Additional file 1: Figure S1. The multiple sequence alignment of TaJAZ proteins. The pink and green lines represent the core sequence of TIFY domain and Jas domain in each of TaJAZ protein. The blank boxes represent the NLS sequence in each of TaJAZ protein. The location of TIFY domain and Jas domain were also marked out in each of TaJAZ protein. Figure S2. Phylogenetic relationship of JAZ between $B$. distachyond and wheat. The BdJAZs in the indicated groups were used as an outgroup to calculate Ka and Ks. Figure S3. The PCR amplified products and subcellular location of TaJAZ7-D, TaJAZ8-D and TaJAZ12-D. (A) lane 1, 3 and 5 represent negative control; lane 2: the PCR amplified product of TaJAZ7-D; lane 4: the PCR amplified product of TaJAZ8-D; lane 6: the PCR amplified product of TaJAZ12-D. (B) the subcellular locations of TaJAZ7-D-GFP, TaJAZ8-D-GFP and TaJAZ12-D-GFP fusion proteins. Scale bars $=20 \mu \mathrm{m}$. (ZIP $3354 \mathrm{~kb})$

Additional file 2: Table S1. The primers used in this study. Table S2. The information of JAZ or JAZ-like genes in different plants. Table S3. The number and composition of cis- acting regulatory elementsof each TaJAZ gene. (ZIP $40 \mathrm{~kb})$

\section{Abbreviations}

ABA: Abscisic acid; GA: Gibberellin; GFP: Green fluorescent protein; IAA: Indole-3-acetic acid; JAZ: Jasmonate-ZIM domain; MeJA: Methyl jasmonate; MYA: Million years ago; ORF: Open reading frame; qRTPCR: Quantitative real-time PCR; SA: Salicylic acid; SLR: Single lens reflex; TGMS: Thermosensitive genic male sterile

\section{Acknowledgements}

We are grateful to Professor Xianchun Xia (Chinese Academy of Agricultural Sciences) for suggestions on this manuscript.

\section{Funding}

This work was financially supported by the National Key Research Project (Grant no. 2016YFD0101601), National Natural Science Foundation of China (Grant no. 31371699), National Science and Technology Support Plan (Grant no. 2013BAD04B01 and 2014BAD01B09), Natural Science Foundation of Beijing (Grant no. 5131001), Special Project of Science and Technology Innovation Ability Construction of BAAFS (Grant no. KJCX20140405) and Shanxi Province Science Foundation for Youths (Grant no. 2015021145).

\section{Availability if data and materials}

All the supporting data are included within the article and its additional files.

\section{Authors' contributions}

LZ and CZ designed the study. YW, LQ and JB performed the experiments, analyzed the data, and drafted the manuscript. PW and WD assisted with bioinformatic analysis and aided in writing the manuscript. SY, GY and FZ aided in performing the experiments. All authors carefully checked and approved this version of the manuscript.

\section{Competing interests}

The authors declare that they have no competing interests.

\section{Consent for publication}

Not applicable.

\section{Author details}

${ }^{1}$ Beijing Engineering Research Center for Hybrid Wheat, Beijing Academy of Agricultural and Forestry Sciences, Beijing 100097, China. ${ }^{2}$ The Municipal Key Laboratory of Molecular Genetics for Hybrid Wheat, Beijing 100097, China. ${ }^{3}$ Institute of Crop Science, Shanxi Academy of Agricultural Sciences, Taiyuan 030031, China. ${ }^{4}$ College of Plant Science and Technology, Beijing University of Agriculture, Beijing 102206, China. ${ }^{5}$ College of Life Sciences, Capital Normal University, Beijing 100048, China.

Received: 2 August 2016 Accepted: 10 February 2017

Published online: 13 February 2017

\section{References}

1. Howe GA, Jander G. Plant immunity to insect herbivores. Annu Rev Plant Biol. 2008:59:41-66.

2. Devoto A, Turner JG. Jasmonate-regulated Arabidopsis stress signaling network. Physiol Plant. 2005;123(2):161-72.

3. Glazebrook J. Contrasting mechanisms of defense against biotrophic and necrotrophic pathogens. Annu Rev Phytopathol. 2005;43:205-27.

4. Gfeller A, Liechti R, Farmer EE. Arabidopsis jasmonate signaling pathway. Sci STKE. 2006:322:cm1.

5. Wasternack C, Stenzel I, Hause B, Hause G, Kutter C, Maucher H, Neumerkel J, Feussner I, Miersch O. The wound response in tomato-role of jasmonic acid. J Plant Physiol. 2006;163(3):297-306.

6. Wasternack C. Jasmonates: An update on biosynthesis, signal transduction and action in plant stress response, growth and development. Ann Bot. 2007;100(4):681-97.

7. Balbi V, Devoto A. Jasmonate signaling network in Arabidopsis thaliana: crucial regulatory nodes and new physiological scenarios. New Phytol. 2008; 177(2):301-18

8. Browse J, Howe GA. New weapons and a rapid response against insect attack. Plant Physiol. 2008;146(3):832-8

9. Schaller F, Schaller A, Stintzi A. Biosynthesis and metabolism of jasmonates. J Plant Growth Regul. 2005;23(3):179-99.

10. Browse J. Jasmonate: an oxylipin signal with many roles in plants. Vitam Horm. 2005:72:431-56.

11. Li L, Zhao Y, MCCaig BC, Wingerd BA, Wang J, Whalon ME, Pichersky E, Howe GA. The tomato homolog of CORONATINE-INSENSITIVE1 is required for the maternal control of seed maturation, jasmonate-signaled defense responses, and glandular trichome development. Plant Cell. 2004;16(1):126-43.

12. Turner JG, Ellis C, Devoto A. The jasmonate signal pathway. Plant Cell. 2002; 14:S153-64.

13. Yan Y, Stolz S, Chételat A, Reymond P, Pagni M, Dubugnon L, Farmer EE. A downstream mediator in the growth repression limb of the jasmonate pathway. Plant Cell. 2007;19(8):2470-83.

14. Giri AP, Wünsche H, Mitra S, Zavala JA, Muck A, Svatos A, Baldwin IT. Molecular interactions between the specialist herbivore Manduca sexta (Lepidoptera, Sphingidae) and its natural host Nicotiana attenuata. VII. Changes in the plant's proteome. Plant Physiol. 2006;142(4):1621-41.

15. Acosta IF, Farmer EE. Jasmonates. Arabidopsis Book. 2010;8:e0129.

16. Gfeller A, Dubugnon L, Liechti R, Farmer EE. Jasmonate biochemical pathway. Sci Signal. 2010;3(109):cm3.

17. Katsir L, Chung HS, Koo AJ, Howe GA. Jasmonate signaling: a conserved mechanism of hormone sensing. Curr Opin Plant Biol. 2008;11(4):428-35.

18. Staswick PE. JAZing up jasmonate signaling. Trends Plant Sci. 2008;13(2):66-71.

19. Chini A, Boter M, Solano R. Plant oxylipins: COI1/JAZs/MYC2 as the core jasmonic acid-signaling module. FEBS J. 2009;276(17):4682-92.

20. Yan J, Zhang C, Gu M, Bai Z, Zhang W, Qi T, Cheng Z, Peng W, Luo H, Nan F, Wang Z, Xie D. The Arabidopsis CORONATINE INSENSITIVE1 protein is a jasmonate receptor. Plant Cell. 2009;21(8):2220-36.

21. Shan X, Yan J, Xie D. Comparison of phytohormone signaling mechanisms. Curr Opin Plant Biol. 2012;15(1):84-91.

22. Melotto M, Mecey C, Niu Y, Chung HS, Katsir L, Yao J, Zeng W, Thines B, Staswick P, Browse J, Howe GA, He SY. A critical role of two positively charged amino acids in the Jas motif of Arabidopsis JAZ proteins in mediating coronatine- and jasmonoyl isoleucine-dependent interactions with the COI1 F-box protein. Plant J. 2008;55(6):979-88. 
23. Chung HS, Howe GA. A critical role for the TIFY motif in repression of jasmonate signaling by a stabilized splice variant of the JASMONATE ZIMdomain protein JAZ10 in Arabidopsis. Plant Cell. 2009;21(1):131-45.

24. Bai $Y$, Meng $Y$, Huang D, Qi Y, Chen M. Origin and evolutionary analysis of the plant-specific TIFY transcription factor family. Genomics. 2011;98(2):128-36.

25. Vanholme B, Grunewald W, Bateman A, Kohchi T, Gheysen G. The tify family previously known as ZIM. Trends Plant Sci. 2007;12(6):239-44.

26. Browse J. Jasmonate passes muster: a receptor and targets for the defense hormone. Annu Rev Plant Biol. 2009;60:183-205.

27. Pauwels L, Barbero GF, Geerinck J, Tilleman S, Grunewald W, Pérez AC, Chico JM, Bossche RV, Sewell J, Gil E, García-Casado G, Witters E, Inzé D, Long JA, De Jaeger G, Solano R, Goossens A. NINJA connects the corepressor TOPLESS to jasmonate signaling. Nature. 2010;464(7289):788-91.

28. Gangappa SN, Prasad VB, Chattopadhyay S. Functional interconnection of MYC2 and SPA1 in the photomorphogenic seedling development of Arabidopsis. Plant Physiol. 2010;154(3):1210-9.

29. Gangappa SN, Chattopadhyay S. MYC2, a bHLH transcription factor, modulates the adult phenotype of SPA1. Plant Signal Behav. 2010;5(12):1650-2.

30. Thines B, Katsir L, Melotto M, Niu Y, Mandaokar A, Liu G, Nomura K, He SY, Howe GA, Browse J. JAZ repressor proteins are targets of the SCF(COI1) complex during jasmonate signaling. Nature. 2007;448(7154):661-5.

31. Chini A, Fonseca S, Fernández G, Adie B, Chico JM, Lorenzo O, GarcíaCasado G, López-Vidriero I, Lozano FM, Ponce MR, Micol JL, Solano R. The JAZ family of repressors is the missing link in jasmonate signaling. Nature. 2007:448(7154):666-71

32. Ye H, Du H, Tang N, Li X, Xiong L. Identification and expression profiling analysis of TIFY family genes involved in stress and phytohormone responses in rice. Plant Mol Biol. 2009;71(3):291-305.

33. Kazan K, Manners JM. JAZ repressors and the orchestration of phytohormone crosstalk. Trends Plant Sci. 2012;17(1):22-31.

34. Demianski AJ, Chung KM, Kunkel BN. Analysis of Arabidopsis JAZ gene expression during Pseudomonas syringae pathogenesis. Mol Plant Pathol. 2012;13(1):46-57.

35. Chung HS, Koo AJ, Gao X, Jayanty S, Thines B, Jones AD, Howe GA Regulation and function of Arabidopsis JASMONATE ZIM-domain genes in response to wounding and herbivory. Plant Physiol. 2008;146(3):952-64.

36. Hori $Y$, Kurotani $K$, Toda $Y$, Hattori T, Takeda S. Overexpression of the JAZ factors with mutated jas domains causes pleiotropic defects in rice spikelet development. Plant Signal Behav. 2014;9(10):e970414.

37. Virmani SS, llyas-Ahmed M. Environment-sensitive genic male sterility (EGMS) in crops. In: Sparks DL, editor. Advance in agronomy. San Diego: Elsevier; 2001. p. 139-95.

38. Ku SJ, Cho KH, Choi YJ, Baek WK, Kim S, Suh HS, Chung YY. Cytological observation of two environmental genic male-sterile lines of rice. Mol Cells. 2001;12(3):403-6.

39. Li Y, Zhao C, Zhang F, Sun H, Sun D. Fertility alteration in the photothermo-sensitive male sterile line BS20 of wheat (Triticum aestivum L.) Euphytica. 2006;151(2):207-13.

40. Xing Q, Ru Z, Li J, Zhou C, Jin D, Sun Y, Wang B. Cloning a second form of adenine phosphoribosyl transferase gene (TAAPT2) from wheat and analysis of its association with thermo-sensitive genic male sterility (TGMS). Plant Sci. 2005;169(1):37-45.

41. Xu C, Liu Z, Zhang L, Zhao C, Yuan S, Zhang F. Organization of actin cytoskeleton during meiosis I in a wheat thermo-sensitive genic male sterile line. Protoplasma. 2013;250(1):415-22.

42. Ma H, Zhang L, Zhao C, Lu X, Zhang F, Yuan S. Effects of methyl-jasmonic acid on the percentage of anther dehiscence and seed set rate of photothermo-sensitive male sterile wheat. Journal of Triticeae Crops. 2011;31(4): 648-52 (in Chinese with English abstract).

43. Singh AP, Pandey BK, Deveshwar P, Narnoliya L, Parida SK, Giri J. JAZ Repressors: Potential Involvement in Nutrients Deficiency Response in Rice and Chickpea. Front Plant Sci. 2015;6:975.

44. Zhang Z, Li X, Yu R, Han M, Wu Z. Isolation, structural analysis, and expression characteristics of the maize TIFY gene family. Mol Genet Genomics. 2015;290(5):1849-58.

45. Zhang L, You J, Chan Z. Identification and characterization of TIFY family genes in Brachypodium distachyon. J Plant Res. 2015;128(6):995-1005.

46. Sheard LB, Tan X, Mao H, Withers J, Ben-Nissan G, Hinds TR, Kobayashi Y, Hsu FF, Sharon M, Browse J, He SY, Rizo J, Howe GA, Zheng N. Jasmonate perception by inositol-phosphate-potentiated COI1-JAZ co-receptor. Nature. 2010;468(7322):400-5.
47. Akhunov ED, Sehgal S, Liang H, Wang S, Akhunova AR, Kaur G, Li W, Forrest KL, See D, Simková H, Ma Y, Hayden MJ, Luo M, Faris JD, Dolezel J, Gill BS. Comparative analysis of syntenic genes in grass genomes reveals accelerated rates of gene structure and coding sequence evolution in polyploid wheat. Plant Physiol. 2013;161(1): 252-65.

48. Tang Z, Zhang L, Yang D, Zhao C, Zheng Y. Cold stress contributes to aberrant cytokinesis during male meiosis I in a wheat thermosensitive genic male sterile line. Plant Cell Environ. 2011;34(3):389-405.

49. Hong H, Xiao H, Yuan H, Zhai J, Huang X. Cloning and characterisation of JAZ gene family in Hevea brasiliensis. Plant Biol (Stuttg). 2015;17(3):618-24.

50. Duan L, Mu X, Li W. Molecular evolution of transcriptional repressor JAZ protein family in jasmonic acid signaling pathway. Chinese Bulletin of Botany. 2013:48(6):623-34 (in Chinese with English abstract).

51. Chico JM, Chini A, Fonseca S, Solano R. JAZ repressors set the rhythm in jasmonate signaling. Curr Opin Plant Biol. 2008;11(5):486-94.

52. Grunewald W, Vanholme B, Pauwels L, Plovie E, Inzé D, Gheysen G, Goossens A. Expression of the Arabidopsis jasmonate signaling repressor JAZ1/TIFY10A is stimulated by auxin. EMBO Rep. 2009;10(8):923-8.

53. Nakashima K, Ito Y, Yamaguchi-Shinozaki K. Transcriptional regulatory networks in response to abiotic stresses in Arabidopsis and grasses. Plant Physiol. 2009;149(1):88-95.

54. Song S, Qi T, Huang H, Ren Q, Wu D, Chang C, Peng W, Liu Y, Peng J, Xie D. The Jasmonate-ZIM domain proteins interact with the R2R3MYB transcription factors MYB21 and MYB24 to affect Jasmonateregulated stamen development in Arabidopsis. Plant Cell. 2011;23(3): $1000-13$

55. Leon-Reyes A, Spoel SH, De Lange ES, Abe H, Kobayashi M, Tsuda S, Millenaar FF, Welschen RA, Ritsema T, Pieterse CM. Ethylene modulates the role of NONEXPRESSOR OF PATHOGENESIS-RELATED GENES1 in cross talk between salicylate and jasmonate signaling. Plant Physiol. 2009;149(4):1797-809.

56. Leon-Reyes A, Van der Does D, De Lange ES, Delker C, Wasternack C, Van Wees SC, Ritsema T, Pieterse CM. Salicylate-mediated suppression of jasmonate-responsive gene expression in Arabidopsis is targeted downstream of the jasmonate biosynthesis pathway. Planta. 2010;232(6): 1423-32.

57. Kunkel BN, Brooks DM. Cross talk between signaling pathways in pathogen defense. Curr Opin Plant Biol. 2002;5(4):325-31.

58. Bari R, Jones JD. Role of plant hormones in plant defence responses. Plant Mol Biol. 2009;69(4):473-88.

59. Quint M, Gray WM. Auxin signaling. Curr Opin Plant Biol. 2006;9(5):448-53.

60. Chen Q, Sun J, Zhai Q, Zhou W, Qi L, Xu L, Wang B, Chen R, Jiang H, Qi J, Li X, Palme K, Li C. The basic helix-loop-helix transcription factor MYC2 directly represses PLETHORA expression during jasmonatemediated modulation of the root stem cell niche in Arabidopsis. Plant Cell. 2011;23(9):3335-52.

61. Aida M, Beis D, Heidstra R, Willemsen V, Blilou I, Galinha C, Nussaume L, Noh YS, Amasino R, Scheres B. The PLETHORA genes mediate patterning of the Arabidopsis root stem cell niche. Cell. 2004;119(1):109-20.

62. Benjamins R, Scheres B. Auxin: the looping star in plant development. Annu Rev Plant Biol. 2008:59:443-65.

63. Li WH, Yang J, Gu X. Expression divergence between duplicate genes. Trends Genet. 2005:21(11):602-7.

64. Seo JS, Joo J, Kim MJ, Kim YK, Nahm BH, Song SI, Cheong JJ, Lee JS, Kim JK, Choi YD. OsbHLH148, a basic helix-loop-helix protein, interacts with OsJAZ proteins in a jasmonate signaling pathway leading to drought tolerance in rice. Plant J. 2011 ; 65(6):907-21.

65. Scott RJ, Spielman M, Dickinson HG. Stamen structure and function. Plant Cell. 2004;16(Suppl):S46-60.

66. Wilson ZA, Song J, Taylor B, Yang C. The final split: the regulation of anther dehiscence. J Exp Bot. 2011;62(5):1633-49.

67. Wang Y, Yuan S, Yuan G, Duan W, Wang P, Liao X, Chen Z, Wang N, Wang Z, Zhang L, Zhao C. Genome-wide identification and analysis of JAZ gene family in Aegilops tauschii. Journal of Triticeae Crops. 2016:36(1):9-17 (in Chinese with English abstract).

68. Qiao L, Zhang X, Han X, Zhang L, Li X, Zhan H, Ma J, Luo P, Zhang W, Cui L, Li X, Chang Z. A genome-wide analysis of the auxin/indole-3-acetic acid gene family in hexaploid bread wheat (Triticum aestivum L.). Front Plant Sci. 2015;6:770. 
69. Yang Z. PAML: a program package for phylogenetic analysis by maximum likelihood. Comput Appl Biosci. 1997;13(5):555-6.

70. Livak KJ, Schmittgen TD. Analysis of relative gene expression data using real-time quantitative PCR and the 2(-Delta Delta C(T)) Method. Methods. 2001;25(4):402-8.

71. Yoo SD, Cho YH, Sheen J. Arabidopsis mesophyll protoplasts: a versatile cell system for transient gene expression analysis. Nat Protoc. 2007;2(7):1565-72.

72. Abel S, Theologis A. Transient transformation of Arabidopsis leaf protoplasts: a versatile experimental system to study gene expression. Plant J. 1994;5(3):421-7.

Submit your next manuscript to BioMed Central and we will help you at every step:

- We accept pre-submission inquiries

- Our selector tool helps you to find the most relevant journal

- We provide round the clock customer support

- Convenient online submission

- Thorough peer review

- Inclusion in PubMed and all major indexing services

- Maximum visibility for your research

Submit your manuscript at www.biomedcentral.com/submit
Biomed Central 